\title{
Near-infrared spectroscopy of five Blue Compact Dwarf galaxies: II Zw 40, Mrk 71, Mrk 930, Mrk 996 and SBS 0335-052E ${ }^{1}$
}

\author{
Yuri I. Izotov \\ Main Astronomical Observatory, Ukrainian National Academy of Sciences, 27 Zabolotnoho \\ str., Kyiv 03680, Ukraine \\ izotov@mao.kiev.ua \\ and \\ Trinh X. Thuan \\ Astronomy Department, University of Virginia, P.O. Box 400325, Charlottesville, VA \\ 22904-4325 \\ txt@virginia.edu
}

\begin{abstract}
We present near-infrared (NIR) spectroscopic observations of five blue compact dwarf (BCD) galaxies, II Zw 40, Mrk 71, Mrk 930, Mrk 996 and SBS 0335052E. The NIR spectra which cover the $0.90 \mu \mathrm{m}-2.40 \mu \mathrm{m}$ wavelength range, show hydrogen, molecular hydrogen, helium, sulfur and iron emission lines. The NIR data for all BCDs have been supplemented by optical spectra. We found the extinction coefficient in all BCDs to be very similar in both the optical and NIR ranges. The NIR hydrogen emission lines do not reveal more star formation than seen in the optical. The same conclusion is reached from Spitzer data concerning the MIR emission lines. This implies that emission-line spectra of low-metallicity BCDs in the $\sim 0.36-25 \mu \mathrm{m}$ wavelength range are emitted by relatively transparent ionized gas. The large extinction derived from the MIR continuum emission in some BCDs implies that the latter arises not from the visible $\mathrm{H}$ II regions themselves, but from locations outside these $\mathrm{H}$ II regions. The $\mathrm{H}_{2}$ emission line fluxes can be accounted for by fluorescence. CLOUDY stellar photoinization models of all BCDs reproduce well the fluxes of most of the observed optical and NIR emission lines, except in Mrk 930 where shock ionization is needed to account for the [Fe II] emission lines. However, some contribution of shock ionization at the level of $\lesssim 10 \%$ that of stellar ionization is required to reproduce the observed fluxes of high ionization species, such as He II $\lambda 0.469 \mu \mathrm{m}$ in the optical range and [O IV] $\lambda 25.89 \mu \mathrm{m}$ in the MIR range.
\end{abstract}


Subject headings: galaxies: abundances — galaxies: irregular — galaxies: ISM - H II regions — galaxies: individual (II Zw 40, Mrk 71, Mrk 930, Mrk 996, SBS 0335-052E) — infrared: ISM

\section{INTRODUCTION}

Blue compact dwarf (BCD) galaxies are the least chemically evolved star-forming galaxies known in the universe (see Thuan 2008, for a review). They have an oxygen abundance in the range $12+\log \mathrm{O} / \mathrm{H}=7.1-8.3$, i.e. $1 / 40-1 / 3$ that of the Sun if the solar abundance of Asplund et al. (2009), $12+\log \mathrm{O} / \mathrm{H}=8.7$, is adopted. They thus constitute excellent nearby laboratories for studying star formation and its interaction with the interstellar medium (ISM) in a very metal-deficient environment, intermediate between the solar-metallicity environment of galaxies such as the Milky Way, and the pristine environment of primordial galaxies. While BCDs have been extensively studied spectroscopically in the optical range, not much work has been done in the near-infrared (NIR) $0.9-2.4 \mu \mathrm{m}$ range. We have thus started a long-term observational program to study the NIR spectroscopic properties of a sample of BCDs chosen to cover a large range of metallicities, with 12 $+\log \mathrm{O} / \mathrm{H}$ ranging from 7.1 to 8.3. The first results of the program, concerning the BCD Mrk $59(12+\log \mathrm{O} / \mathrm{H}=8.0)$, have been discussed by Izotov et al. (2009). Here, we present new $J, H$ and $K$ spectroscopic observations of five more BCDs, with $12+\log \mathrm{O} / \mathrm{H}$ ranging from 7.29 to 8.10 .

In this new list, only two BCDs have had previous NIR observations. II Zw 40 has been studied by Joy \& Lester (1988), Moorwood \& Oliva (1988), Mouri et al. (1990), Vanzi et al. (1996), Coziol et al. (2001) and Vanzi et al. (2008), while SBS 0335-052E has been observed by Vanzi et al. (2000). However, all these observations covered a more restricted wavelength range, including only either the $H$ band and/or the $K$ band. The other three BCDs, Mrk 71, Mrk 930 and Mrk 996, have not been observed spectroscopically in the NIR before. The general characteristics of the five objects are given in Table 1. With advances in NIR detector sensitivities, and with the commisionning in 2008 of the TripleSpec spectrograph at the Apache Point Observatory (APO) 3.5-meter telescope2, we can obtain NIR spectra with a simultaneous coverage of the $J H K$ bands and considerably

\footnotetext{
${ }^{1}$ Tables $3-7$ are presented only in the electronic edition of the Journal.

${ }^{2}$ The Apache Point Observatory 3.5-meter telescope is owned and operated by the Astrophysical Research Consortium.
} 
more signal-to-noise. These improved observations will allow us to study in more detail the physical conditions in BCDs, and check for their dependence on metallicity. We will be able to compare the extinction in the optical and NIR and search for hidden star formation. We will also be able to study the excitation mechanisms of line emission of ionized species in the $\mathrm{H}$ II region, and search for molecular hydrogen emission.

In addition to the NIR observations, we have also obtained optical spectra of the same objects, except for Mrk 930, in the wavelength range $0.36-0.97 \mu \mathrm{m}$. These were taken so to have an overlap with the NIR spectra in the $0.90-0.97 \mu \mathrm{m}$ range, which allows us to match the two types of spectra and reduce the uncertainties due to different apertures and/or varying weather conditions.

We describe the 3.5m APO observations for the five new BCDs in Section 2, In Section 3. we discuss their extinction, both in the optical and NIR ranges, and in the MIR range using Spitzer data, the excitation mechanisms for molecular hydrogen and iron emission in the NIR range, and CLOUDY modeling of the H II region. We summarize our findings in Section 4 .

\section{OBSERVATIONS}

\subsection{Near-infrared observations}

NIR spectra of the BCDs II Zw 40, Mrk 71, Mrk 930, Mrk 996 and SBS 0335-052E were obtained with the $3.5 \mathrm{~m}$ APO telescope in conjunction with the TripleSpec spectrograph on the night of 2009 November 25. TripleSpec (Wilson et al. 2004) is a cross-dispersed NIR spectrograph that provides simultaneous continuous wavelength coverage from 0.90 to 2.46 $\mu \mathrm{m}$ in five spectral orders during a single exposure. A $1 .{ }^{\prime \prime} 1 \times 43^{\prime \prime}$ slit was used, resulting in a resolving power of 3500 .

Three A0V standard stars, HD 16286, HD 23504, HD 212170 were observed for flux calibration and correction for telluric absorption. Spectra of Ar comparison arcs were obtained on the same date for wavelength calibration. Since all observed targets are smaller than the length of the slit, the nod-on-slit technique was used to acquire the sky spectrum. Objects were observed by nodding between two positions A and B along the slit, following the ABBA sequence, and with an integration time of 200 s or 300 s at each position. The journal of the NIR observations is given in Table 2. We show there the observation date, the exposure time, the extraction aperture of the one-dimensional spectrum and the average airmass. 
We carried out the reduction of the data according to the following procedures. The two-dimensional spectra were first cleaned for cosmic ray hits using the IRAF 3 routine CRMEDIAN. Then all A and B frames were separately coadded and the resulting B frame was subtracted from the resulting A frame. Finally, the (negative) spectrum at position B was adjusted to the (positive) spectrum at position $\mathrm{A}$ and subtracted from it. The same reduction scheme was applied to the standard stars.

We then use the IRAF routines IDENTIFY, REIDENTIFY, FITCOORD, TRANSFORM to perform wavelength calibration and correction for distortion and tilt for each frame. For all galaxies, a one-dimensional spectrum was extracted from the two-dimensional frames using the APALL IRAF routine, within a $1^{\prime \prime} .1 \times 6^{\prime \prime}$ rectangular aperture so as to include the brightest star-forming regions. The redshift of our objects range from 0.00036 for Mrk 71 to 0.0183 for Mrk 930, so that $1^{\prime \prime}$ corresponds to $7 \mathrm{pc}$ for the nearest object and $350 \mathrm{pc}$ for the furthest object, adopting a Hubble constant of $75 \mathrm{~km} \mathrm{~s}^{-1} \mathrm{Mpc}^{-1}$. For the nearer objects, II Zw 40, Mrk 71 and Mrk 996, the slit contains a single stellar cluster which dominates in the line emission. Thus, in II Zw 40 the slit is centered on the compact super-star cluster (SSC) A (Vanzi et al. 2008), in Mrk 71 on the brightest compact cluster A (Thuan \& Izotov 2005), and in Mrk 996 on the central cluster (Thuan et al. 1996). All these stellar clusters are unresolved on HST/WFPC2 images. For the furthest galaxies, SBS 0335-052E and Mrk 930, the slit is also centered on the brightest cluster, but contains several other stellar clusters. The HST images of Mrk 930 and SBS 0335-052E are shown in Malkan et al. (1998) and Thuan et al. (1997), respectively. For SBS 0335-052E, the slit is oriented at a position angle of $-30^{\circ}$ and includes not only SSCs 1 and 2, which dominate the line emission, but also SSCs 3-6. The spectrum of of Mrk 930 (its southern knot) was obtained at a position angle of $+238^{\circ}$. Because of the compact structure of SBS 0335-052E and Mrk 930, we have not attempted to extract spectra of individual clusters and have used the integrated spectra. For these two furthest objects, the larger linear sizes of the slit also mean that a larger area of the weakly ionized and neutral ISM around the stellar clusters is included in the slit, a fact which will be relevant in our modelization of the line emission of the low-ionization and neutral species (Section 3.3) and in our discussion of the MIR extinction (Section 3.6).

Flux calibration and correction for telluric absorption were performed by first multiplying the one-dimensional spectrum of each galaxy by the synthetic absolute spectral distribution of the brightest standard star HD 23504, smoothed to the same spectral resolution,

\footnotetext{
${ }^{3} \mathrm{IRAF}$ is the Image Reduction and Analysis Facility distributed by the National Optical Astronomy Observatory, which is operated by the Association of Universities for Research in Astronomy (AURA) under cooperative agreement with the National Science Foundation (NSF).
} 
and then by dividing the result by the observed one-dimensional spectrum of the same star. Since there does not exist any published absolute spectral energy distribution of standard stars, we have derived the one of HD 23504 by scaling the synthetic absolute spectral energy distribution of the star Vega ( $\alpha$ Lyrae), of similar spectral type A0V, to the brightness of HD 23504.

The resulting one-dimensional flux-calibrated and redshift-corrected NIR spectra of all five BCDs are shown in Figs. 1]- 5 ,

\subsection{Optical observations}

To have a more complete physical picture of the extinction and of the ionization mechanisms in our five BCDs, we have supplemented the NIR observations with optical ones. The optical observations of all galaxies, with the exception of Mrk 930, were obtained with the 3.5 $\mathrm{m}$ APO telescope in the course of several nights during the period November 2008 - February 2010. For Mrk 930, we have used the optical spectrum obtained by Izotov \& Thuan (1998).

The $3.5 \mathrm{~m}$ APO observations were made with the Dual Imaging Spectrograph (DIS) in both the blue and red wavelength ranges. In the blue range, we use the B400 grating with a linear dispersion of $1.83 \times 10^{-4} \mu \mathrm{m} /$ pix and a central wavelength of $0.44 \mu \mathrm{m}$, while in the red range we use the R300 grating with a linear dispersion $2.31 \times 10^{-4} \mu \mathrm{m} / \mathrm{pix}$ and a central wavelength of $0.75 \mu \mathrm{m}$. The above instrumental set-up gave a spatial scale along the slit of $0^{\prime \prime} .4$ pixel $^{-1}$, a spectral range $\sim 0.36-0.97 \mu \mathrm{m}$ and a spectral resolution of $7 \times 10^{-4}$ $\mu \mathrm{m}$ (FWHM). Several Kitt Peak IRS spectroscopic standard stars were observed for flux calibration. Spectra of He-Ne-Ar comparison arcs were obtained at the beginning or the end of each night for wavelength calibration. The journal of the optical observations is given in Table 2. As for Mrk 930, the acquisition of its optical spectrum in the wavelength range $0.36-0.74 \mu \mathrm{m}$ is described in Izotov \& Thuan (1998).

The two-dimensional optical spectra were bias subtracted and flat-field corrected using IRAF. We then use the IRAF software routines IDENTIFY, REIDENTIFY, FITCOORD, TRANSFORM to perform wavelength calibration and correction for distortion and tilt for each frame. One-dimensional spectra were then extracted from each frame using the APALL routine. The extraction apertures for the one-dimensional optical spectra are shown in Table 2. The areas of these optical extraction apertures are generally similar to those of the NIR extraction apertures. An exception is Mrk 930, for which the area of the optical extraction aperture is $\sim 2$ times that of the NIR extraction aperture. The sensitivity curve was obtained by fitting with a high-order polynomial the observed spectral energy distribution of standard 
stars. Then sensitivity curves obtained from observations of different stars during the same night were averaged. The flux deviation from curve to curve is less than $1-2 \%$.

We have used the optical spectra to derive the physical conditions and oxygen abundances of all five BCDs, following the procedures described by Izotov et al. (2006a). The derived oxygen abundances are shown in Table 1.

The NIR and optical spectra of all BCDs, except for Mrk 930, overlap in the $0.90-$ $0.97 \mu \mathrm{m}$ wavelength range, allowing us to match the fluxes of strong emission lines that are common in the two types of spectra. Specifically, we have adjusted the optical and NIR spectra so that the flux of the strongest line in the overlapping region, [S III] $0.953 \mu \mathrm{m}$, is the same in both spectra. We have thus multiplied the NIR spectra of II Zw 40, Mrk 71, Mrk 996 and SBS 0335-052E by factors of 1.27, 1.17, 1.10 and 1.09, respectively. The multiplying factors are close to unity, as expected from the similarities of the areas of the optical and NIR extraction apertures. The deviations from unity can be caused by the slighly differing extraction apertures, non-perfect pointing and varying weather conditions (transparency, seeing). They can also be caused by uncertainties in the flux calibration of the NIR spectra introduced by uncertainties in the absolute spectral energy distribution (SED) of the NIR standard star HD 23504. As discussed in Sect. 2.1, this SED is not known and was obtained by scaling the synthethic NIR spectrum of Vega.

Additional uncertainties are introduced by the ionization structure of the $\mathrm{H}$ II regions, i.e. differences in the spatial distribution of various ions. In particular, hydrogen line emission is more concentrated to the center of the star-forming region than the $[\mathrm{S}$ III] emission. We find that the FWHM of the $\mathrm{H} \alpha$ emission along the spatial axis of our objects is $\sim 20 \%$ smaller than that of the [S III] $\lambda 0.953 \mu \mathrm{m}$ emission. The ionization structure uncertainties are difficult to estimate as the observations were obtained with different slit widths, 1".5 for optical spectra and $1^{\prime \prime}$. 1 for NIR ones. We have attempted to minimize these uncertainties by extracting one-dimensional spectra within apertures with similar areas.

For Mrk 930, we have scaled the NIR spectrum by a factor of 1.73 so that the $\mathrm{Br} \gamma / \mathrm{H} \beta$ flux ratio is equal to the theoretical recombination value, after correcting the emission line fluxes for interstellar extinction and underlying stellar absorption derived from the optical spectrum. This scaling factor agrees nicely with the ratio of the areas of the apertures used for the NIR and optical observations of Mrk 930. The combined redshift-corrected optical and NIR spectra for all BCDs are shown in Figs. 6-10, Gaps in the NIR spectra correspond to regions of strong absorption by telluric lines. While the scaling of the NIR spectrum of Mrk 930 is more uncertain, it seems justified by the fact that NIR SED extends smoothly the optical SED, without any abrupt discontinuity (Fig. 8). 


\section{RESULTS AND DISCUSSION}

Emission-line fluxes in both the optical and NIR ranges were measured by using the SPLOT routine in IRAF. The errors of the line fluxes were calculated from the photon statistics in the non-flux-calibrated spectra. They do not include systematic errors such as deviations of the standard star SED from the Vega SED, different apertures used in the optical and NIR observations and varying weather conditions. Those are difficult to estimate as discussed in Sect. 2.2. The observed fluxes $F(\lambda)$ of emission lines derived from the adjusted optical and NIR spectra are shown in the second columns of Tables 3 - 7 , They are normalized to the observed $\mathrm{H} \beta$ flux, $F(\mathrm{H} \beta)$, shown at the end of each table, and multiplied by a factor of 100 .

How do our observations compare with previous ones of the same objects? II $\mathrm{Zw}$ 40 was observed by Joy \& Lester (1988) and Coziol et al. (2001) in the $K$ band, and by Moorwood \& Oliva (1988), Vanzi et al. (1996) and Vanzi et al. (2008) in the $H$ and $K$ bands. Joy \& Lester (1988) (3"'6 aperture), Vanzi et al. (1996) (2".4 aperture) and Coziol et al. (2001) (2-3" aperture) obtained a flux of the $\operatorname{Br} \gamma$ emission line $F(\operatorname{Br} \gamma)$ in the range (3.4-3.9) $\times 10^{-14} \mathrm{erg} \mathrm{s}^{-1} \mathrm{~cm}^{-2}$, slightly larger than our value of $3.1 \times 10^{-14} \mathrm{erg} \mathrm{s}^{-1}$ $\mathrm{cm}^{-2}\left(1^{\prime \prime} .1 \times 6^{\prime \prime}\right.$ aperture). On the other hand, Moorwood \& Oliva (1988) found $F(\operatorname{Br} \gamma)=$ $6.7 \times 10^{-14} \mathrm{erg} \mathrm{s}^{-1} \mathrm{~cm}^{-2}$ in a $6^{\prime \prime} \times 6^{\prime \prime}$ aperture, while Vanzi et al. (2008) derived $F(\operatorname{Br} \gamma)=$ $1.0 \times 10^{-14} \mathrm{erg} \mathrm{s}^{-1} \mathrm{~cm}^{-2}$ within a circular aperture of $1^{\prime \prime}$ in radius. SBS $0335-052 \mathrm{E}$ has been observed in the NIR only by Vanzi et al. (2000) who derived $F(\operatorname{Br} \gamma)=1.5 \times 10^{-15} \mathrm{erg} \mathrm{s}^{-1}$ $\mathrm{cm}^{-2}$ in an aperture of $1^{\prime \prime} \times 1^{\prime \prime} .5$, lower than our value of $2.4 \times 10^{-15} \mathrm{erg} \mathrm{s}^{-1} \mathrm{~cm}^{-2}$. All observations of II $\mathrm{Zw} 40$ and SBS 0335-052E appear to be consistent in the sense that the $\operatorname{Br} \gamma$ fluxes obtained with apertures having similar areas are close to each other while those obtained with smaller apertures are lower.

The presence of many emission lines in the optical and NIR spectra (Figs. 1-10 and Tables 3-7) allows us to study extinction, $\mathrm{H}_{2}$ and [Fe II] emission and excitation mechanisms in all five galaxies. We discuss these issues in the following sections.

\subsection{Optical and NIR extinction and hidden star formation}

In previous optical-NIR studies of BCDs, except for our own work on Mrk 59 Izotov et al. 2009), JHK spectra have been observed separately, and there has been no wavelength overlap between the optical and NIR spectra. This introduces uncertainties, due to the use of generally different apertures in the optical and NIR observations, and due to the adjusting of the continuum levels of the NIR spectra obtained separately in different orders. Because our 
NIR spectra have been obtained simultaneously over the whole $J H K$ wavelength range and because it is possible to directly match the optical and NIR spectra using common emission line fluxes (except for Mrk 930), these adjusting uncertainties are mostly eliminated. This allows us to directly compare the optical and NIR extinctions and search for any hidden extinction at longer wavelengths.

In the second column of Tables 3- 7, we list the observed fluxes $F(\lambda)$ of the emission lines in both the optical and NIR spectra. In the third column of the same Tables are shown the fluxes $I(\lambda)$, corrected for underlying stellar absorption (for hydrogen lines) and extinction (for all lines). The extinction coefficient $C(\mathrm{H} \beta)$ and equivalent width $\mathrm{EW}(\mathrm{abs})$ of hydrogen lines derived from the decrement of hydrogen Balmer lines in the optical spectrum are listed in the footnotes to Tables 3- 7. They are used to correct the emission line fluxes in both the optical and NIR ranges, adopting the extinction curve of Cardelli et al. (1989) and $R(V)=$ $A(V) / E(B-V)=3.2$, where $A(V)$ and $E(B-V)$ are respectively the $V$ band extinction and the $B-V$ reddening. The value $R(V)=3.2$ is typical for the diffuse interstellar medium in the Galaxy. With this value of $R(V)$, then $C(\mathrm{H} \beta)=1.47 \times E(B-V)=0.46 \times A(V)$.

However, the dust composition in the low-metallicity interstellar medium in our BCDs can be different from that in the Galaxy, resulting in a different extinction curve. Indeed, Rocca-Volmerange et al. (1981) found that the UV extinction curve in the Small Magellanic Cloud (SMC) is significantly steeper than that in the Galaxy, indicating the presence of a larger fraction of small dust grains in the SMC. To reproduce the SMC extinction curve in the UV range, the value of $R(V)$ should be smaller than 3.2. However, differences in the extinction curves with different $R(V) \mathrm{s}$ are much smaller in the optical and NIR ranges. For example, if we adopt $R(V)=2.5$, corresponding to $C(\mathrm{H} \beta)=1.17 \times E(B-V)=0.47 \times A(V)$, then $C(\mathrm{H} \beta)$ is decreased by $\sim 10 \%$ and the flux of the $\operatorname{Br} \gamma$ emission line relative to $\mathrm{H} \beta$ is increased only by $\sim 2-3 \%$, much smaller than the errors given in Tables $3-7$ for that line. Therefore, in the following, we will use the extinction-corrected emission-line fluxes obtained with $R(V)=3.2$.

The corrected fluxes of hydrogen lines in the third column of the same Tables are derived, assuming that the difference between the observed and theoretical hydrogen Balmer decrements is solely due to extinction and underlying stellar absorption. However, collisional excitation of hydrogen lines may play a role, resulting in a steepening of the observed Balmer decrement. Following Luridiana (2009), we assume that the fraction of the $\mathrm{H} \alpha$ emission due to collisional excitation is $5 \%$, while that for $\mathrm{H} \beta, \mathrm{H} \gamma$ and $\mathrm{H} \beta$ emission is $3 \%$. These values should be considered as upper limits for collisional excitation. A possible exception is Mrk 996 with a very high-density H II region (Thuan et al. 2008), where collisional excitation could be higher. Subtracting the collisional contribution from the $\mathrm{H} \alpha, \mathrm{H} \beta, \mathrm{H} \gamma$ and $\mathrm{H} \delta$ 
emission lines and correcting all lines for extinction and hydrogen lines for underlying stellar absorption, we obtain the emission line fluxes listed in the fourth column of Tables [3 - 7 .

In the second to sixth columns of Table 8 , we show the extinction-corrected fluxes $I(\lambda)$ of the strongest hydrogen lines in the optical and NIR spectra for all observed galaxies. For comparison, the seventh column of Table 8 shows the theoretical recombination flux ratios,

calculated by Hummer \& Storey (1987) for case B, with an electron temperature $T_{e}=15000$ $\mathrm{K}$ and an electron number density $N_{e}=100 \mathrm{~cm}^{-3}$. It can be seen that there is a very good agreement between the corrected and theoretical recombination values of the optical and NIR line fluxes. We have shown in Table 9 the extinction coefficients $C(\mathrm{H} \beta)$ derived from the ratio of the flux of each individual hydrogen line to the $\mathrm{H} \beta$ flux. They were derived from the equation

$$
\frac{I(\lambda)}{I(\mathrm{H} \beta)}=\frac{F(\lambda)}{F(\mathrm{H} \beta)} \frac{E W(\lambda)+E W(a b s)}{E W(\lambda)} \frac{E W(\mathrm{H} \beta)}{E W(\mathrm{H} \beta)+E W(a b s)} \times 10^{f(\lambda) C(\mathrm{H} \beta)},
$$

where $f(\lambda)$ is the value of the normalized reddening curve from Cardelli et al. (1989) at the wavelength $\lambda$, adopting $A(V) / E(B-V)=3.2, F$ and $I$ are respectively the observed and theoretical fluxes, $\mathrm{EW}(\lambda)$ and $\mathrm{EW}(\mathrm{H} \beta)$ are equivalent widths of emission lines, and $\mathrm{EW}(\mathrm{abs})$ is the equivalent width of the absorption hydrogen lines. We adopt EW(abs) =

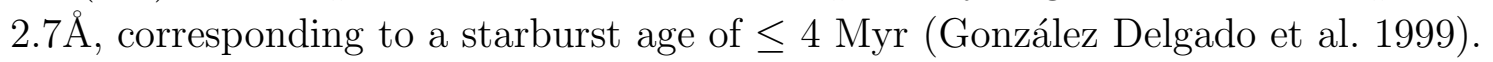

Inspection of Table 9 shows that, for each object, the extinction coefficients $C(\mathrm{H} \beta)$ derived from different hydrogen lines agree within the errors and do not show any systematic trend. This implies that the same extinction coefficient $C(\mathrm{H} \beta)$ [or extinction $A(V)$ ] holds over the whole $0.36-2.40 \mu \mathrm{m}$ wavelength range. The fact that $A(V)$ does not increase at longer wavelengths implies that there is no hidden star formation in the NIR as compared to in the optical range. This appears to be a general result for BCDs (e.g. Vanzi et al. 2000, 2002; Izotov et al. 2009). This is true even in the extreme case of the BCD SBS 0335-052E where observations of the far-infrared (FIR) continuum imply a very large extinction $[A(V)$ $\sim 15-20 \mathrm{mag}$ ] and three times as much hidden star formation in the MIR than in the visible (Thuan et al. 1999; Houck et al. 2004). We will explore the MIR extinction in section 3.6.

\section{2. $\mathrm{H}_{2}$ emission}

Molecular hydrogen lines do not originate in the $\mathrm{H}$ II region, but in neutral molecular clouds. In the NIR, they are excited by two mechanisms. The first one is the thermal mechanism consisting of collisions between neutral species (e.g., $\mathrm{H}, \mathrm{H}_{2}$ ), resulting from large-scale shocks driven by powerful stellar winds, supernova remnants or molecular cloud collisions. 
The second one is the fluorescent mechanism due to absorption of ultraviolet photons. It is known that these two mechanisms excite mostly different roto-vibrational levels of $\mathrm{H}_{2}$. By comparing the observed line ratios with those predicted by models such as those calculated by Black \& van Dishoeck (1987), it is possible to discriminate between the two processes. In particular, line emission from the vibrational level $v=2$ and higher vibrational levels are virtually absent in collisionally excited spectra, while they are relatively strong in fluorescent spectra.

$\mathrm{H}_{2}$ emission lines are detected in the NIR spectra of all our objects (see Figs. 1-5).

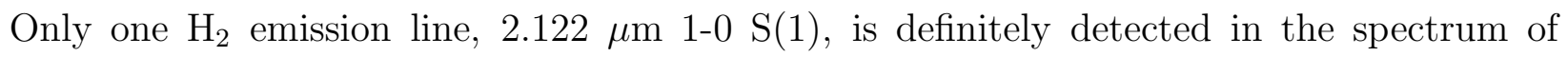
SBS 0335-052E. Another line, $2.248 \mu \mathrm{m} \mathrm{2-1} \mathrm{S(1),} \mathrm{is} \mathrm{marginally} \mathrm{detected,} \mathrm{although} \mathrm{it} \mathrm{is}$ clearly present in the spectrum of Vanzi et al. (2000). Three, five, thirteen and fourteen $\mathrm{H}_{2}$ lines are respectively detected in the NIR spectra of Mrk 930, Mrk 996, II Zw 40 and Mrk 71. The fluxes of the $\mathrm{H}_{2}$ lines relative to that of the strongest $2.122 \mu \mathrm{m} \mathrm{1-0} \mathrm{S(1)} \mathrm{line}$ are shown in Table 10. In the last two columns are shown the theoretical ratios calculated by Black \& van Dishoeck (1987) in the cases of fluorescent and collisional excitation. It is seen that the observed line flux ratios are in agreement with those predicted for fluorescent excitation, despite the rather high errors in the flux ratios. Our finding is in agreement with the conclusions of Vanzi et al. (2000) for SBS 0335-052E, of Vanzi et al. (2008) for II $\mathrm{Zw} 40$ and of Izotov et al. (2009) for Mrk 59, where it was also found that the fluorescence process is the main excitation mechanism of $\mathrm{H}_{2}$ lines. This conclusion appears to be common for BCDs with high-excitation spectra.

\subsection{CLOUDY stellar photoionization modeling of the $\mathrm{H}$ II region}

We next examine the excitation mechanisms of the NIR emission lines arising in the H II regions of our BCDs. For this purpose, we have constructed stellar photoionization models for the H II regions using the CLOUDY code (version 07.02.01) of Ferland (1996) and Ferland et al. (1998). We list in Table 11 the input parameters of these models. The first row gives $Q(\mathrm{H})$, the logarithm of the number of ionizing photons per second, calculated from the $\mathrm{H} \beta$ luminosity with the distance given in Table 1 . For the determination of the $\mathrm{H} \beta$ luminosity, we have taken the $\mathrm{H} \beta$ fluxes from large aperture spectroscopic and photometric observations. We thus adopt the $\mathrm{H} \beta$ fluxes to be $3.5 \times 10^{-13} \mathrm{erg} \mathrm{s}^{-1} \mathrm{~cm}^{-2}$ for II Zw 40 (Lagos et al. 2007), $2.1 \times 10^{-12} \mathrm{erg} \mathrm{s}^{-1} \mathrm{~cm}^{-2}$ for Mrk 71 (Moustakas \& Kennicutt 2006) and $2.5 \times 10^{-13} \mathrm{erg} \mathrm{s}^{-1} \mathrm{~cm}^{-2}$ for Mrk 930 (Moustakas \& Kennicutt 2006). These fluxes were then corrected for extinction, as derived from the spectroscopic observations. We also adopt the extinction-corrected $\mathrm{H} \beta$ fluxes of $6.6 \times 10^{-13} \mathrm{erg} \mathrm{s}^{-1} \mathrm{~cm}^{-2}$ for Mrk 996 (Thuan et al. 
2008) and of $1.8 \times 10^{-13} \mathrm{erg} \mathrm{s}^{-1} \mathrm{~cm}^{-2}$ for SBS 0335-052E (Izotov et al. 2006b).

The other rows of Table 11 list $T_{\text {eff }}$, the effective temperature of stellar radiation, and $N_{e}$, the electron number density, assumed to be constant with radius for all objects, except for Mrk 996, for which we have adopted the number density radial distribution of Thuan et al. (2008). The fourth row gives $f$, the filling factor. The remaining input parameters are the number ratios of different species to hydrogen, derived mainly from the spectroscopic observations in this paper, except for the carbon and silicon abundances. There are no strong emission lines of these last two species in the optical and NIR ranges. We have therefore calculated their abundances, using the mean relations of $\mathrm{C} / \mathrm{O}$ and $\mathrm{Si} / \mathrm{O}$ vs. oxygen abundance derived for low-metallicity H II regions by Garnett et al. (1995a, $\underline{b}$ ). For the ionizing stellar radiation, we have adopted a Kurucz (1979) stellar atmosphere model with $T_{\text {eff }}=50000 \mathrm{~K}$. We have also experimented with other forms of the stellar ionizing radiation such as those given by Starburst99 models (Leitherer et al. 1999) or by models based on the Geneva stellar evolutionary tracks with heavy element mass fractions $Z=0.001-0.004$ (Meynet et al. 1994). In all cases, we were able to reproduce well most of the observed line intensities by varying only slightly the input parameters. Thus our results are robust and do not depend sensitively on a particular adopted grid of models.

Most of the input parameters in Table 11, e.g. $Q(\mathrm{H})$ and chemical composition, are constrained by observations and are thus kept unchanged. This is not the case for the filling factor $f$ which is therefore varied until the best agreement is found between the observed and predicted line intensities. The CLOUDY-predicted fluxes of the emission lines are shown in the fifth column of Tables 3 - 7. Comparison of the extinction-corrected observed and predicted emission-line fluxes in both the optical and NIR ranges shows that, in general, the agreement between the observations and the CLOUDY predictions is satisfactory. This is especially true for the strong hydrogen, neutral helium and forbidden lines of doubly ionized species. The agreement however is not as good for emission lines of singly ionized and neutral species which arise in the outer parts of H II regions or even beyond the ionized hydrogen regions (e.g. the $[\mathrm{S}$ II $]$ emission lines). For example, the line intensities of the $[\mathrm{S} \mathrm{II}] \lambda \lambda$ 6717,6731 emission lines are underpredicted by a factor of 2 for the nearer BCDs (Mrk 71 and II Zw 40) and by a factor of 3-4 for the more distant ones (SBS 0335-052 and Mrk 930). The larger disagreement factor for the more distant BCDs is probably due to the fact that their extraction apertures are larger in linear size and include more of the weakly ionized and neutral ISM than the apertures of the nearer BCDs.

Some part of the disagreement can be caused by our too simplistic modeling with the CLOUDY code. For all galaxies but Mrk 996, we have adopted a spherically symmetric $\mathrm{H}$ II region model with a single compact central ionizing source and an uniform density distri- 
bution. In reality, our galaxies are likely to contain several non-symmetric and non-uniform $\mathrm{H}$ II regions with different ionization parameters which contribute in different proportions to the excitation of high- and low-ionization species in the integrated spectra. With the exception of Mrk 996, we do not have enough observational data to produce more complex and refined models of the H II regions. For the sole case of Mrk 996, we have adopted a non-uniform radial density distribution (the one dervived by Thuan et al. 1996, 2008), and there is indeed a much better agreement between the observed and predicted line intensities, particularly for the $[\mathrm{S}$ II $] \lambda \lambda 6717,6731$ emission lines.

More sophisticated modeling will certainly improve the overall agreement between observations and models. However, these improvements will not change the main conclusion that $\mathrm{H}$ II region models including only stellar photoionization as an ionizing source can account for most of the observed line fluxes, both in the optical and NIR ranges. For the great majority of the lines, no additional excitation mechanism such as shocks from stellar winds and supernova remnants is needed. This situation is again similar to that in the BCDs II Zw 40 and Mrk 59, where Vanzi et al. (2008) and Izotov et al. (2009) found that the ISM is mainly photoexcited by stars. However, we note that while some He I emission lines in the spectrum of Mrk 996 are reproduced reasonably well, the modeled fluxes of the He I $\lambda 0.707 \mu \mathrm{m}$ and $1.083 \mu \mathrm{m}$ emission lines are several times larger than those observed. These two lines are very sensitive to collisional and fluorescent excitation. In the very high-density H II region of Mrk 996 (Thuan et al. 2008), the He I $\lambda 1.083 \mu \mathrm{m}$ is optically thick. This may be a reason why this line has an asymmetric profile (see inset in Fig. (4). Therefore, a detailed treatment of the radiative transfer in this line and of the collisional excitation of $\mathrm{He}$ I states is needed.

\subsection{The contribution of shock photoionization}

There are other exceptions. One such exception is the He II $\lambda 0.469 \mu \mathrm{m}$ nebular emission line. It is detected in the optical spectra of three BCDs, Mrk 71, Mrk 930 and SBS 0335-052E, but cannot be reproduced by the photoionized $\mathrm{H}$ II region models with only stellar radiation as the ionizing source. In the two remaining BCDs, II Zw 40 and Mrk 996, this emission line is also detected, but it is broad, implying that it is produced by expanding envelopes of Wolf-Rayet stars, and not by nebular emission. To reproduce the observed nebular He II $\lambda 0.469 \mu \mathrm{m}$ emission-line flux, a harder ionizing radiation is required. Therefore, for all BCDs (except for II Zw 40 and Mrk 996), we have also calculated models of H II regions with a constant number density, which include ionization radiation by fast shocks, in addition to the stellar ionizing radiation. We vary the number of ionizing photons produced by fast 
shocks until the model prediction best matches the observed flux of the nebular He II $\lambda 0.469$ $\mu \mathrm{m}$ emission line. We adopt the spectral distribution of the ionizing radiation to be that of

a shock with a velocity of $500 \mathrm{~km} \mathrm{~s}^{-1}$ (Allen et al. 2008). For best agreement, we found the number of ionizing photons from fast shocks to be respectively $2.5 \%, 4 \%$ and $13 \%$ the number of ionizing stellar photons for Mrk 71, Mrk 930 and SBS 0335-052E. In the last column of Tables 4, 5 and 7, we show the emission-line fluxes predicted by combined models including both stellar and shock ionizing radiation. These models reproduce well the observed nebular He II $\lambda 0.469 \mu \mathrm{m}$ emission line fluxes. They also increase the relative fluxes of the lowionization species, such as [O I] $\lambda 0.630$ and $[\mathrm{S}$ II] $\lambda 0.672,0.673 \mu \mathrm{m}$ emission lines, making them agree better with the observations. On the other hand, the relative line fluxes of the high-ionization species such as [Ne III] $\lambda 0.387$, and [S III] $\lambda 0.9533 \mu \mathrm{m}$ are little changed. This is probably because the shock-produced harder ionizing radiation penetrates more efficiently into the outer layers of the H II region, as compared to the softer stellar ionizing radiation. This results in more efficient heating of the gas and excitation of the low-ionization species.

\section{5. [Fe II] line emission}

The [Fe II] $\lambda 1.257$ and $\lambda 1.643 \mu \mathrm{m}$ emission lines have often been used as indicators of the importance of shock excitation relative to that of photoionization (Moorwood \& Oliva 1988; Oliva et al. 1990, 2001). All of our galaxies either show one of them or both. In the Galaxy, the intensities of these lines are considerably smaller in regions where photoionization dominates than in those where shock excitation dominates, such as in SN remnants. For example, the ratio of the [Fe II] $\lambda 1.643 \mu \mathrm{m}$ line to $\operatorname{Br} \gamma$ is 0.06 in the Orion nebula, while it is more than 30 in Galactic SN remnants (Moorwood \& Oliva 1988; Oliva et al. 1990).

The [Fe II] $\lambda 1.257 \mu \mathrm{m}$ and $\lambda 1.643 \mu \mathrm{m}$ lines to $\operatorname{Br} \gamma$ ratios have the very low values of $\sim 0.05-0.1$ in II Zw 40, Mrk 71 and SBS 0335-052E, similar to the value of 0.055 found by Izotov et al. (2009) in Mrk 59, and to the one in the Orion nebula. However, in the two other galaxies, Mrk 930 and Mrk 996, which have lower excitation H II regions, the [Fe $\mathrm{II}] / \mathrm{Br} \gamma$ ratios are considerably higher, with values $\sim 0.5$, but still much lower than the value of $>30$ in Galactic SN remnants. Does this mean that SN shock excitation does not play a role in all our BCDs?

Examination of Table 12 shows that, even without any contribution of shock excitation from SNe, the stellar photoionization CLOUDY models overpredict the [Fe II] $\lambda 1.257$ and $\lambda 1.643 \mu \mathrm{m}$ line fluxes by factors of $\sim 1.5-3$. An exception is Mrk 930, where the extinctioncorrected [Fe II] line fluxes are a factor of $\sim 2$ greater than the modeled ones. If shock ionizing radiation is included (last column of Table [12), then the modeled [Fe II] emission 
line fluxes are more comparable to the observed ones in Mrk 930. However, they are much higher than the observed fluxes in other galaxies. It appears that, for $\mathrm{H}$ II regions in II Zw 40, Mrk 71, Mrk 996 and SBS 0335-052E, there is no need to invoke any shock ionizing radiation. However, shocks may play an important role in the excitation of [Fe II] emission lines in Mrk 930.

The overprediction of the [Fe II] fluxes in the four BCDs other than Mrk 930 may be due to aperture effects. The small apertures used in the optical and NIR observations (the slit widths are respectively $1^{\prime \prime} .5$ and $1^{\prime \prime} .1$ ) include only the inner parts of the $\mathrm{H}$ II region while singly ionized iron is mainly located in its outer parts. This conclusion appears to be supported by the fact that the discrepancy factor is largest for the nearest BCD, Mrk 71, at a distance of $3.4 \mathrm{Mpc}$, while it is lower for more distant galaxies, with distances larger than $10 \mathrm{Mpc}$. The aperture effect is the smallest for the most distant BCD, Mrk 930. Izotov et al. (2009) have proposed another explanation for the BCD Mrk 59, at a distance of 10.7 Mpc. They argued that the overprediction by a factor of $\sim 2$ of the [Fe II] 1.257 and $1.643 \mu \mathrm{m}$ emission line intensities by the CLOUDY stellar ionization model for this BCD can be explained by the depletion of iron atoms onto dust grains (Oliva et al. 2001; Izotov et al. 2006a). This depletion process is likely to be most efficient in the outer zones of the $\mathrm{H}$ II region where the [Fe II] emission lines arise. These have less intense ionizing radiation as compared to the inner zones where the ionizing radiation is more intense and where the [Fe III] optical emission lines arise. It is likely that both aperture effects and depletion of iron onto dust play a role in accounting for the discrepancies between the modeled and observed [Fe II] fluxes.

\subsection{Comparison with mid-infrared observations: MIR extinction}

We have shown above that the observed relative line flux ratios in both the optical and NIR ranges in all our objects can be satisfactorily accounted for with the same extinction coefficient $C(\mathrm{H} \beta)$ [or the same extinction $A(V)$ ]. In other words, there is no more hidden star formation in the NIR range as compared to in the optical range in regions traced by emission lines, i.e. in ionized gas regions. A question then arises: would we see more hidden star formation at longer wavelengths, in the mid-infrared (MIR) range?

To investigate the extinction in the MIR range, we have used the published Spitzer MIR emission line fluxes of Wu et al. (2006) and O'Halloran et al. (2006) for II Zw 40, of O'Halloran et al. (2006) for Mrk 930, of Thuan et al. (2008) for Mrk 996, and of Houck et al. (2004) for SBS 0335-052E. Spitzer observations of Mrk 71 exist but have not yet been published. Since the Spitzer spectra were obtained within significantly larger apertures 
as compared to the optical and NIR spectra, we have normalized the MIR emission-line fluxes to the optical and NIR fluxes by multiplying them by the ratios of the extinctioncorrected optical $\mathrm{H} \beta$ fluxes listed in Tables 3-7 to those obtained from large aperture spectroscopic observations or from photometric observations. Thus, for II Zw 40 and Mrk 930 we have adopted the $\mathrm{H} \beta$ fluxes of Lagos et al. (2007) and Moustakas \& Kennicutt (2006), respectively. These fluxes were then corrected for extinction with the extinction coefficient $C(\mathrm{H} \beta)$ derived in this paper from the optical spectra. For Mrk 996 and SBS 0335-052E, we have adopted respectively the extinction-corrected $\mathrm{H} \beta$ fluxes of Thuan et al. (2008) and Izotov et al. (2006a). The multiplicative factors are respectively $0.32,0.27,0.31$, and 0.50 for II Zw 40, Mrk 930, Mrk 996 and SBS 0335-052E. No extinction correction has been applied to the MIR data as it is presumably small in this wavelength range.

The normalized MIR emission line fluxes are shown in Table 13 together with the flux predictions by the CLOUDY models discussed in Section 3.3 for the optical and NIR ranges. Comparison of the MIR observed fluxes with those predicted by the pure stellar ionizing radiation model shows agreement to within a factor of 2 . The disagreement is the worst for the low ionization lines [Ne II] $\lambda 12.81 \mu \mathrm{m}$ in II Zw 40 and [Fe II] $\lambda 25.99 \mu \mathrm{m}$ in II Zw 40 and Mrk 930, the fluxes of which are predicted to be considerably smaller than the observed ones. Furthermore, the presence of the high-ionization emission line [O IV] $\lambda 25.89 \mu \mathrm{m}$ in Mrk 996 cannot be accounted for by a pure stellar ionizing radiation model (Thuan et al. 2008). The high observed flux of [Fe II] $\lambda 25.99 \mu \mathrm{m}$ in Mrk 930 cannot be explained by shock excitation, but it can be understood if we note that neutral iron has an ionization potential smaller than that of hydrogen. Therefore, singly ionized iron may exist in weakly ionized cool interstellar medium, outside the $\mathrm{H}$ II regions, and contribute to the [Fe II] $\lambda 25.99 \mu \mathrm{m}$ emission, a contribution which is not accounted for in CLOUDY models. As for the highionization [O IV] $\lambda 25.89 \mu \mathrm{m}$ emission line in Mrk 996, Thuan et al. (2008) found that a CLOUDY model where the fraction of ionizing photons from shocks is only $3 \%$ fits well all the MIR emission-lines of Mrk 996, including [O IV] $\lambda 25.89 \mu \mathrm{m}$. We give the predictions of that model for Mrk 996 in Table 13. The agreement within a factor of 2 between the observed fluxes of the MIR emission-lines and the predicted ones of CLOUDY models, based on the extinction-corrected optical emission lines, implies that there is not considerably more hidden star formation seen in the MIR range, as compared to in the optical and NIR ranges. This implies that, in low-metallicity BCDs, the H II regions with emission lines detected in the MIR range are in general relatively transparent, so they are also seen in the optical and NIR ranges.

At first glance, this conclusion appears to be in flagrant disagreement with the findings of Thuan et al. (1999) and Houck et al. (2004) who inferred highly obscured regions in SBS 0335-052E, with an $A(V) \sim 15$ mag, from modeling of the MIR continuum emission 
produced by warm dust. Based on these observations, Thuan et al. (1999) concluded that some $75 \%$ of the star formation in SBS 0335-052E is hidden. These two apparently discrepant results can be reconciled if the region where resides the warm dust responsible for the MIR emission is different from the ionized gas region where the emission lines arise. Studies of the H II regions in the Milky Way (e.g., Lebouteiller et al. 2007) show a good correlation between e.g. the [S IV] $\lambda 10.51 \mu \mathrm{m}$ emission line flux and the warm dust continuum, implying that the warm dust resides in the ionized medium. However, the physical conditions in SBS 0335-052E are very different from those in the Milky Way H II regions. In particular, star formation in SBS 0335-052E occurs in several compact SSCs containing several thousand low-metallicity O stars (e.g., Thuan et al. 1997; Johnson et al. 2009), responsible for a very high UV flux in the H II regions around the SSCs. It is doubtful whether dust grains can survive in such an extreme environment. Furthermore, if the warm dust is in the same region as the ionized gas, we would expect an excess of the MIR emission line fluxes as compared to the predicted ones. No such excess is found, at least for the [Ne III] $\lambda 15.55$ $\mu \mathrm{m}$ emission line (Table 13). The dust is likely located in the neutral gas that surrounds the compact H II regions and which does not contribute to the observed MIR emission lines. In other words, the MIR emission lines and continuum arise in different regions, the first ones in relatively transparent $\mathrm{H}$ II regions and the second one in highly extincted regions surrounding the $\mathrm{H}$ II regions.

\section{SUMMARY AND CONCLUSIONS}

We present here near-infrared (NIR) spectroscopic observations of five blue compact dwarf (BCD) galaxies, II Zw 40, Mrk 71, Mrk 930, Mrk 996 and SBS 0335-052E, obtained with the $3.5 \mathrm{~m}$ APO telescope. The NIR data have been supplemented by optical spectra obtained with the $3.5 \mathrm{~m}$ APO and the $2.1 \mathrm{~m}$ KPNO telescopes, resulting in a total wavelength coverage of $0.36-2.46 \mu \mathrm{m}$. The overlap in the $0.90-0.97 \mu \mathrm{m}$ wavelength range allows to adjust the continuum levels of the NIR spectra to the optical ones by insuring that the flux of the $[\mathrm{S} \mathrm{III}] \lambda 0.953 \mu \mathrm{m}$ emission line is the same in both types of spectra.

We have arrived at the following conclusions:

1. In all BCDs, the extinction coefficient $C(\mathrm{H} \beta)=0.46 \times A(V)$ derived in the optical is the same as the one derived in the NIR for the ionized gas regions traced by emission lines. The NIR emission lines do not probe more extinct regions with hidden star formation as compared to the optical emission lines. Using Spitzer MIR data, we have also found that the extinction $A(V)$ inferred from MIR observations is similar to the one derived from optical and NIR observations. Thus, the emission-line spectra of low-metallicity BCDs in the whole 
$\sim 0.36-25 \mu \mathrm{m}$ wavelength range originates in relatively transparent $\mathrm{H}$ II regions. The large extinction deduced from the MIR continuum emission in the BCD SBS 0335-052E probably arises from neutral regions surrounding the $\mathrm{H}$ II regions.

2. We have detected molecular hydrogen emission lines in the NIR spectra of all BCDs. Comparison of the observed fluxes with modeled ones suggests that the main excitation mechanism of $\mathrm{H}_{2}$ emission in all BCDs is fluorescence.

3. A CLOUDY model with a pure stellar ionizing radiation reproduces well the observed fluxes of most emission lines in both optical and NIR ranges. Shocks are likely present in the $\mathrm{H}$ II region, but play a minor role in the ionization, at the level of $10 \%$ or less, except for high-ionization emission lines, such as He II $\lambda 0.469 \mu \mathrm{m}$ in the optical range and [O IV] $\lambda 25.89 \mu \mathrm{m}$ in the MIR range.

4. CLOUDY stellar and shock ionizing models show that the [Fe II] $\lambda 1.257$ and $\lambda 1.643$ $\mu \mathrm{m}$ emission lines, often used as SN shock indicators in low-excitation high-metallicity starburst galaxies, cannot play such a role in high-excitation low-metallicity $\mathrm{H}$ II regions, such as those in the BCDs II Zw 40, Mrk 71, Mrk 996 and SBS 0335-052E. However, we find that shocks could be important contributors to the emission of [Fe II] lines in Mrk 930.

The 3.5 APO time was available thanks to a grant from the Frank Levinson Fund of

the Silicon Valley Community Foundation to the Astronomy Department of the University of Virginia. Y.I.I. is grateful to the staff of the Astronomy Department at the University of Virginia for their warm hospitality. T.X.T acknowledges the support of NASA.

\section{REFERENCES}

Allen, M. G., Groves, B. A., Dopita, M. A., Sutherland, R. S., \& Kewley, L. J. 2008, ApJS, 178,20

Asplund, M., Grevesse, N., Sauval, J., \& Scott, P. 2009, ARAA, 47, 481

Black, J. H., \& van Dishoeck, E. F. 1987, ApJ, 322, 412

Cardelli, J. A., Clayton, G. C., \& Mathis, J. S. 1989, ApJ, 345, 245

Coziol, R., Doyon, R., \& Demers, S. 2001, MNRAS, 325, 1081

Ferland, G. J. 1996, Hazy: A brief Introduction to CLOUDY, Univ. Kentucky Phys. Dept. Int. Rep. 
Ferland, G. J., Korista, K. T., Verner, D. A., Ferguson, J. W., Kingdon, J. B., \& Verner, E. M. 1998, PASP, 110, 761

Garnett, D. R., et al. 1995a, ApJ, 443, 64

Garnett, D. R., et al. 1995b, ApJ, 449, 77

González Delgado, R. M., Leitherer, C., \& Heckman, T. M. 1999, ApJS, 125, 489

Houck, J. R. et al. 2004, ApJS, 154, 211

Hummer, D. G., \& Storey, P. J. 1987, MNRAS, 224, 801

Izotov, Y. I., \& Thuan, T. X. 1998, ApJ, 500, 188

Izotov, Y. I., \& Thuan, T. X. 1999, ApJ, 511, 639

Izotov, Y. I., Stasińska, G., Meynet, G., Guseva, N. G., \& Thuan T. X. 2006a, A\&A, 448, 955

Izotov, Y. I., Schaerer, D., Blecha, A., Royer, F., Guseva, N. G., \& North, P. 2006b, A\&A, 459,71

Izotov, Y. I., Thuan, T. X., \& Wilson, J. C. 2009, ApJ, 703, 1984

Johnson, K. E., Hunt, L. K., \& Reines, A. E. 2009, AJ, 137, 3788

Joy, M., \& Lester, D. F. 1988, ApJ, 331, 145

Kurucz, R. L. 1979, ApJS, 40, 1

Lagos, P., Telles, E., \& Melnick, J. 2007, A\&A, 476, 89

Lebouteiller, V., Brandl, B., Bernard-Salas, J., Devost, D., \& Houck, J. R. 2007, ApJ, 665, 390

Leitherer, C., et al. 1999, ApJS, 123, 3

Luridiana, V. 2009, Ap\&SS, 324, 361

Malkan, M. A., Gorjian, V., \& Tam, R. 1998, ApJS, 117, 25

Meynet, G., Maeder, A., Schaller, G., Schaerer, D., \& Charbonnel, C. 1992, A\&A, 103, 97

Moorwood, A. F. M., \& Oliva, E. 1988, A\&A, 203, 278 
Mouri, H., Nishida, M., Taniguchi, Y., \& Kawara, K. 1990, ApJ, 360, 55

Moustakas, J., \& Kennicutt, R. C., Jr. 2006, ApJS, 164, 81

O’Halloran, B., Satyapal, S., \& Dudik, R. P. 2006, ApJ, 641, 795

Oliva, E., Moorwood, A. F. M., \& Danziger, I. J. 1990, A\&A, 240, 453

Oliva, E., et al. 2001, A\&A, 369, L5

Rocca-Volmerange, B., Prévot, L., Ferlet, R., Lequeux, J., \& Prévot-Burnichon, M. L. 1981, A\&A, 99, L5

Thuan, T. X., 2008, in Low-metallicity star formation: from the first stars to dwarf galaxies, ed. L. Hunt, S.C. Madden, \& R. Schneider (Cambridge: Cambridge Univ. Press), 348

Thuan, T. X., \& Izotov, Y. I. 2005, ApJ, 627, 739

Thuan, T. X., Izotov, Y. I., \& Lipovetsky, V. A. 1996, ApJ, 463, 120

Thuan, T. X., Izotov, Y. I., \& Lipovetsky, V. A. 1997, ApJ, 477, 661

Thuan, T. X., Sauvage, M., \& Madden, S. 1999, ApJ, 516, 783

Thuan, T. X., Hunt, L. K., \& Izotov, Y. I. 2008, ApJ, 689, 897

Vanzi, L., Rieke, G. H., Martin, C. L., \& Shields, J. C. 1996, ApJ, 466, 150

Vanzi, L., Hunt, L. K., Thuan, T. X., \& Izotov, Y. I. 2000, A\&A, 363, 493

Vanzi, L., Hunt, L. K., \& Thuan, T. X. 2002, A\&A, 390, 481

Vanzi, L., Cresci, G., Telles, E., \& Melnick, J. 2008, A\&A, 486, 393

Wilson, J. C., et al. 2004, SPIE, 5492, 1295

Wu, Y., et al. 2006, ApJ, 639, 157

This preprint was prepared with the AAS LATEX macros v5.2. 
Table 1. General characteristics of galaxies

\begin{tabular}{lccccc}
\hline \hline \multicolumn{1}{c}{ Object } & R.A. (J2000.0) & Dec. (J2000.0) & Redshift & Distance $^{\mathrm{a}}$ & $12+\log \mathrm{O} / \mathrm{H}^{\mathrm{b}}$ \\
\hline II Zw 40 & 055542.6 & +032332 & 0.00263 & 10.4 & 8.00 \\
Mrk 71 & 072842.8 & +691121 & 0.00036 & 3.4 & 7.85 \\
Mrk 930 & 233158.3 & +285650 & 0.01830 & 77.4 & 8.08 \\
Mrk 996 & 012735.5 & -061936 & 0.00541 & 21.8 & 8.10 \\
SBS 0335-052E & 033744.0 & -050240 & 0.01348 & 53.8 & 7.29 \\
\hline
\end{tabular}

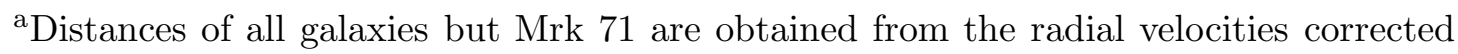
for the Virgo infall and are taken from the NASA/IPAC Extragalactic Database (NED). The distance of Mrk 71 is from Thuan \& Izotov (2005).

$\mathrm{b}$ this paper.

Table 2. Journal of observations

\begin{tabular}{|c|c|c|c|c|}
\hline Object & Date & Exposure (s) & $\begin{array}{l}\text { Extraction } \\
\text { aperture }^{\mathrm{a}}\end{array}$ & Airmass \\
\hline \multicolumn{5}{|c|}{ Near-infrared observations } \\
\hline II Zw 40 & 25 Nov. 2009 & $2000(4 \times 300+4 \times 200)$ & $1^{\prime \prime} 1 \times 6^{\prime \prime}$ & 1.7 \\
\hline Mrk 71 & 25 Nov. 2009 & $1200(4 \times 300)$ & $1^{\prime \prime} 1 \times 6^{\prime \prime}$ & 1.8 \\
\hline Mrk 930 & 25 Nov. 2009 & $1200(4 \times 300)$ & $1^{\prime \prime} 1 \times 6^{\prime \prime}$ & 1.0 \\
\hline Mrk 996 & 25 Nov. 2009 & $2400(8 \times 300)$ & $1^{\prime \prime} 1 \times 6^{\prime \prime}$ & 1.7 \\
\hline SBS $0335-052 \mathrm{E}$ & 25 Nov. 2009 & $3600(12 \times 300)$ & $1^{\prime \prime} 1 \times 6^{\prime \prime}$ & 1.7 \\
\hline \multicolumn{5}{|c|}{ Optical observations } \\
\hline II Zw 40 & 07 Feb. 2010 & 1200 & $1^{\prime \prime} .5 \times 4^{\prime \prime}$ & 1.2 \\
\hline Mrk 71 & 20 Nov. 2009 & 660 & $1^{\prime \prime} .5 \times 4^{\prime \prime}$ & 1.3 \\
\hline Mrk 930 & 16 Dec. 1996 & 3600 & $2^{\prime \prime} \times 6^{\prime \prime} .9$ & 1.0 \\
\hline Mrk 996 & 06 Nov. 2008 & 1200 & $1^{\prime \prime} .5 \times 4^{\prime \prime}$ & 1.7 \\
\hline SBS 0335-052E & 20 Nov. 2009 & 1200 & $1^{\prime \prime} .5 \times 4^{\prime \prime}$ & 1.3 \\
\hline
\end{tabular}

${ }^{\text {a} E x t r a c t i o n ~ a p e r t u r e ~ f o r ~ t h e ~ o n e-d i m e n s i o n a l ~ s p e c t r u m . ~}$ 
Table 3. Fluxes of optical and NIR emission lines in II Zw 40

\begin{tabular}{|c|c|c|c|c|}
\hline ION & $100 \times F(\lambda) / F(\mathrm{H} \beta)^{\mathrm{a}}$ & $100 \times I(\lambda) / I(\mathrm{H} \beta)^{\mathrm{b}}$ & $100 \times I(\lambda) / I(\mathrm{H} \beta)^{\mathrm{c}}$ & $\frac{\text { CLOUDY }}{\text { stars }}$ \\
\hline \multicolumn{5}{|c|}{ a) Optical spectrum } \\
\hline $0.373[\mathrm{O} \quad \mathrm{II}]$ & $23.87 \pm 0.66$ & $59.06 \pm 2.03$ & $55.59 \pm 2.03$ & 53.28 \\
\hline $0.380 \mathrm{H} 10$ & $3.30 \pm 0.39$ & $7.53 \pm 1.29$ & $7.49 \pm 1.29$ & 5.68 \\
\hline 0.384 H9 & $3.91 \pm 0.34$ & $8.70 \pm 1.27$ & $8.67 \pm 1.28$ & 7.70 \\
\hline 0.387 [Ne III $]$ & $28.90 \pm 0.56$ & $61.52 \pm 1.94$ & $62.24 \pm 1.94$ & 60.73 \\
\hline $0.389 \mathrm{He} \mathrm{I+H8}$ & $8.52 \pm 0.34$ & $18.14 \pm 1.39$ & $18.22 \pm 1.39$ & 17.84 \\
\hline $0.389[\mathrm{Ne} \mathrm{III}]+\mathrm{H} 7$ & $17.70 \pm 0.41$ & $35.54 \pm 1.56$ & $35.89 \pm 1.57$ & 34.62 \\
\hline $0.410 \mathrm{H} \delta$ & $17.89 \pm 0.37$ & $32.61 \pm 1.36$ & $32.04 \pm 1.33$ & 26.34 \\
\hline $0.434 \mathrm{H} \gamma$ & $32.89 \pm 0.54$ & $49.49 \pm 1.48$ & $48.92 \pm 1.47$ & 47.42 \\
\hline $0.436[\mathrm{O} \mathrm{III}]$ & $8.67 \pm 0.22$ & $12.77 \pm 0.70$ & $13.04 \pm 0.71$ & 11.34 \\
\hline $0.447 \mathrm{He} \mathrm{I}$ & $2.83 \pm 0.17$ & $3.81 \pm 0.38$ & $3.90 \pm 0.38$ & 3.95 \\
\hline $0.471[$ Ar IV $]+$ He I & $2.68 \pm 0.14$ & $2.98 \pm 0.30$ & $3.07 \pm 0.30$ & 2.77 \\
\hline $0.474[\mathrm{Ar}$ IV $]$ & $1.18 \pm 0.13$ & $1.29 \pm 0.20$ & $1.33 \pm 0.21$ & 1.69 \\
\hline $0.486 \mathrm{H} \beta$ & $100.00 \pm 1.49$ & $100.00 \pm 1.59$ & $100.00 \pm 1.60$ & 100.00 \\
\hline $0.496[\mathrm{O}$ III $]$ & $258.52 \pm 3.79$ & $241.50 \pm 3.72$ & $249.58 \pm 3.84$ & 226.58 \\
\hline $0.501[\mathrm{O}$ III $]$ & $797.24 \pm 11.6$ & $721.72 \pm 10.6$ & $746.46 \pm 11.0$ & 682.00 \\
\hline 0.588 He I & $13.75 \pm 0.24$ & $8.06 \pm 0.35$ & $8.43 \pm 0.36$ & 9.99 \\
\hline $0.630[\mathrm{O} \mathrm{I}]$ & $2.40 \pm 0.10$ & $1.20 \pm 0.13$ & $1.26 \pm 0.13$ & 0.68 \\
\hline 0.631 [S III $]$ & $2.98 \pm 0.11$ & $1.49 \pm 0.14$ & $1.56 \pm 0.14$ & 1.70 \\
\hline $0.636[\mathrm{O} \mathrm{I}]$ & $1.14 \pm 0.10$ & $0.56 \pm 0.09$ & $0.59 \pm 0.09$ & 0.22 \\
\hline $0.656 \mathrm{H} \alpha$ & $622.51 \pm 9.07$ & $283.94 \pm 4.56$ & $283.94 \pm 4.56$ & 282.41 \\
\hline 0.658 [N II] & $9.54 \pm 0.17$ & $4.32 \pm 0.23$ & $4.55 \pm 0.23$ & 2.33 \\
\hline $0.668 \mathrm{He}$ I & $7.26 \pm 0.14$ & $3.18 \pm 0.19$ & $3.35 \pm 0.20$ & 2.72 \\
\hline $0.672[\mathrm{~S}$ II $]$ & $10.72 \pm 0.19$ & $4.63 \pm 0.23$ & $4.88 \pm 0.24$ & 2.64 \\
\hline $0.673[\mathrm{~S}$ II] & $10.20 \pm 0.18$ & $4.39 \pm 0.22$ & $4.62 \pm 0.23$ & 2.00 \\
\hline $0.707 \mathrm{He} \mathrm{I}$ & $13.13 \pm 0.21$ & $5.01 \pm 0.23$ & $5.30 \pm 0.24$ & 4.20 \\
\hline 0.714 [Ar III] & $21.55 \pm 0.34$ & $8.03 \pm 0.29$ & $8.49 \pm 0.30$ & 8.91 \\
\hline $0.728 \mathrm{He} \mathrm{I}$ & $2.18 \pm 0.09$ & $0.77 \pm 0.08$ & $0.82 \pm 0.09$ & 0.68 \\
\hline $0.732[\mathrm{O}$ II] & $4.33 \pm 0.11$ & $1.51 \pm 0.12$ & $1.60 \pm 0.12$ & 0.98 \\
\hline $0.733[\mathrm{O}$ II $]$ & $3.79 \pm 0.14$ & $1.32 \pm 0.11$ & $1.40 \pm 0.12$ & 0.78 \\
\hline 0.775 [Ar III $]$ & $6.20 \pm 0.15$ & $1.86 \pm 0.12$ & $1.98 \pm 0.13$ & 2.15 \\
\hline $0.805[\mathrm{Cl} \mathrm{IV}]$ & $1.26 \pm 0.08$ & $0.34 \pm 0.05$ & $0.37 \pm 0.05$ & 0.39 \\
\hline
\end{tabular}


Table 3-Continued

\begin{tabular}{|c|c|c|c|c|}
\hline ION & $100 \times F(\lambda) / F(\mathrm{H} \beta)^{\mathrm{a}}$ & $100 \times I(\lambda) / I(\mathrm{H} \beta)^{\mathrm{b}}$ & $100 \times I(\lambda) / I(\mathrm{H} \beta)^{\mathrm{c}}$ & $\frac{\text { CLOUDY }}{\text { stars }}$ \\
\hline $0.847 \mathrm{~Pa} 17$ & $1.32 \pm 0.09$ & $0.33 \pm 0.09$ & $0.35 \pm 0.09$ & 0.29 \\
\hline $0.850 \mathrm{~Pa} 16$ & $2.30 \pm 0.11$ & $0.56 \pm 0.09$ & $0.59 \pm 0.10$ & 0.35 \\
\hline $0.855 \mathrm{~Pa} 15$ & $2.19 \pm 0.10$ & $0.53 \pm 0.09$ & $0.56 \pm 0.09$ & 0.45 \\
\hline $0.860 \mathrm{~Pa} 14$ & $3.25 \pm 0.11$ & $0.77 \pm 0.09$ & $0.81 \pm 0.10$ & 0.98 \\
\hline $0.867 \mathrm{~Pa} 13$ & $3.02 \pm 0.11$ & $0.70 \pm 0.09$ & $0.74 \pm 0.10$ & 1.02 \\
\hline 0.875 Pa12 & $4.76 \pm 0.13$ & $1.07 \pm 0.10$ & $1.14 \pm 0.10$ & 1.20 \\
\hline $0.886 \mathrm{~Pa} 11$ & $5.11 \pm 0.15$ & $1.12 \pm 0.10$ & $1.19 \pm 0.10$ & 1.48 \\
\hline $0.901 \mathrm{~Pa} 10$ & $9.72 \pm 0.21$ & $2.05 \pm 0.12$ & $2.19 \pm 0.12$ & 1.92 \\
\hline 0.907 [S III] & $62.59 \pm 0.95$ & $12.97 \pm 0.33$ & $13.93 \pm 0.35$ & 13.30 \\
\hline $0.923 \mathrm{~Pa} 9$ & $10.48 \pm 0.23$ & $2.12 \pm 0.12$ & $2.27 \pm 0.13$ & 2.58 \\
\hline 0.953 [S III] & $141.62 \pm 2.14$ & $26.92 \pm 0.59$ & $28.97 \pm 0.63$ & 32.98 \\
\hline $0.955 \mathrm{~Pa} \epsilon$ & $19.30 \pm 0.50$ & $3.67 \pm 0.16$ & $3.94 \pm 0.17$ & 3.65 \\
\hline \multicolumn{5}{|c|}{ b) Near infrared spectrum } \\
\hline 0.907 [S III $]$ & $59.60 \pm 8.72$ & $12.34 \pm 1.81$ & $13.26 \pm 1.95$ & 13.30 \\
\hline 0.953 [S III] & $141.62 \pm 7.73$ & $26.92 \pm 1.52$ & $28.97 \pm 1.63$ & 32.98 \\
\hline $0.955 \mathrm{~Pa} \epsilon$ & $16.26 \pm 0.96$ & $3.09 \pm 0.20$ & $3.32 \pm 0.22$ & 3.65 \\
\hline $1.003 \mathrm{He} \mathrm{I}$ & $1.84 \pm 0.17$ & $0.32 \pm 0.04$ & $0.35 \pm 0.04$ & 0.12 \\
\hline $1.005 \mathrm{~Pa} \delta$ & $26.89 \pm 1.47$ & $4.71 \pm 0.28$ & $5.07 \pm 0.30$ & 5.45 \\
\hline $1.029[\mathrm{~S} \mathrm{II}]$ & $0.55 \pm 0.10$ & $0.09 \pm 0.02$ & $0.10 \pm 0.02$ & 0.08 \\
\hline $1.032[\mathrm{~S} \mathrm{II}]$ & $0.49 \pm 0.06$ & $0.08 \pm 0.02$ & $0.09 \pm 0.02$ & 0.11 \\
\hline $1.083 \mathrm{He} \mathrm{I}$ & $190.47 \pm 10.4$ & $29.93 \pm 1.69$ & $32.39 \pm 1.83$ & 26.94 \\
\hline $1.091 \mathrm{He} \mathrm{I}$ & $2.75 \pm 0.17$ & $0.43 \pm 0.04$ & $0.46 \pm 0.05$ & 0.28 \\
\hline $1.094 \mathrm{Pa \gamma}$ & $47.21 \pm 2.57$ & $7.33 \pm 0.42$ & $7.92 \pm 0.45$ & 8.74 \\
\hline $1.197 \mathrm{He} \mathrm{I}$ & $1.74 \pm 0.11$ & $0.24 \pm 0.03$ & $0.26 \pm 0.03$ & 0.19 \\
\hline $1.207 \mathrm{H}_{2} 3-1 \mathrm{~S}(2)$ & $0.14 \pm 0.01$ & $0.02 \pm 0.01$ & $0.02 \pm 0.01$ & $\cdots$ \\
\hline $1.233 \mathrm{H}_{2} 3-1 \mathrm{~S}(1)$ & $0.30 \pm 0.04$ & $0.04 \pm 0.02$ & $0.04 \pm 0.02$ & $\cdots$ \\
\hline $1.238 \mathrm{H}_{2} 2-0 \mathrm{Q}(1)$ & $0.43 \pm 0.06$ & $0.06 \pm 0.03$ & $0.06 \pm 0.03$ & $\cdots$ \\
\hline $1.253 \mathrm{He} \mathrm{I}$ & $1.57 \pm 0.10$ & $0.21 \pm 0.03$ & $0.23 \pm 0.03$ & 0.25 \\
\hline $1.257[\mathrm{Fe} \mathrm{II}]$ & $2.13 \pm 0.13$ & $0.28 \pm 0.03$ & $0.30 \pm 0.03$ & 0.39 \\
\hline $1.279 \mathrm{He} \mathrm{I}$ & $6.68 \pm 0.37$ & $0.86 \pm 0.06$ & $0.94 \pm 0.07$ & 0.69 \\
\hline $1.282 \mathrm{~Pa} \beta$ & $94.07 \pm 5.12$ & $12.13 \pm 0.69$ & $13.19 \pm 0.75$ & 15.55 \\
\hline $1.311 \mathrm{H}_{2} 4-2 \mathrm{~S}(1)$ & $0.28 \pm 0.04$ & $0.04 \pm 0.02$ & $0.04 \pm 0.02$ & $\ldots$ \\
\hline
\end{tabular}


Table 3-Continued

\begin{tabular}{|c|c|c|c|c|}
\hline ION & $100 \times F(\lambda) / F(\mathrm{H} \beta)^{\mathrm{a}}$ & $100 \times I(\lambda) / I(\mathrm{H} \beta)^{\mathrm{b}}$ & $100 \times I(\lambda) / I(\mathrm{H} \beta)^{\mathrm{c}}$ & $\frac{\text { CLOUDY }}{\text { stars }}$ \\
\hline $1.314 \mathrm{H}_{2} 3-1 \mathrm{Q}(1)$ & $0.31 \pm 0.01$ & $0.04 \pm 0.02$ & $0.04 \pm 0.02$ & $\cdots$ \\
\hline $1.500 \mathrm{Br} 24$ & $0.72 \pm 0.06$ & $0.08 \pm 0.02$ & $0.09 \pm 0.02$ & 0.20 \\
\hline $1.504 \mathrm{Br} 23$ & $0.79 \pm 0.06$ & $0.09 \pm 0.02$ & $0.10 \pm 0.02$ & 0.22 \\
\hline $1.509 \mathrm{Br} 22$ & $1.12 \pm 0.07$ & $0.13 \pm 0.02$ & $0.14 \pm 0.03$ & 0.23 \\
\hline 1.514 Br21 & $1.18 \pm 0.07$ & $0.14 \pm 0.03$ & $0.15 \pm 0.03$ & 0.09 \\
\hline 1.519 Br20 & $1.45 \pm 0.08$ & $0.17 \pm 0.03$ & $0.18 \pm 0.03$ & 0.10 \\
\hline 1.526 Br19 & $0.98 \pm 0.06$ & $0.11 \pm 0.02$ & $0.12 \pm 0.02$ & 0.11 \\
\hline 1.535 $\mathrm{Br} 18$ & $1.56 \pm 0.09$ & $0.18 \pm 0.03$ & $0.19 \pm 0.03$ & 0.12 \\
\hline 1.544 Br17 & $1.53 \pm 0.09$ & $0.17 \pm 0.03$ & $0.18 \pm 0.03$ & 0.14 \\
\hline 1.556 $\mathrm{Br} 16$ & $2.25 \pm 0.13$ & $0.25 \pm 0.03$ & $0.27 \pm 0.03$ & 0.17 \\
\hline 1.570 $\mathrm{Br} 15$ & $2.18 \pm 0.12$ & $0.24 \pm 0.03$ & $0.26 \pm 0.03$ & 0.22 \\
\hline 1.588 Br14 & $2.63 \pm 0.15$ & $0.29 \pm 0.03$ & $0.31 \pm 0.03$ & 0.47 \\
\hline $1.601 \mathrm{H}_{2} 6-4 \mathrm{Q}(1)$ & $0.24 \pm 0.03$ & $0.03 \pm 0.01$ & $0.03 \pm 0.01$ & $\cdots$ \\
\hline $1.611 \mathrm{Br} 13$ & $3.73 \pm 0.21$ & $0.40 \pm 0.04$ & $0.43 \pm 0.04$ & 0.49 \\
\hline $1.641 \mathrm{Br} 12$ & $4.25 \pm 0.23$ & $0.45 \pm 0.04$ & $0.49 \pm 0.04$ & 0.57 \\
\hline $1.643[\mathrm{Fe} \mathrm{II}]$ & $2.05 \pm 0.11$ & $0.22 \pm 0.03$ & $0.24 \pm 0.03$ & 0.38 \\
\hline $1.681 \mathrm{Br} 11$ & $6.10 \pm 0.33$ & $0.63 \pm 0.05$ & $0.69 \pm 0.05$ & 0.71 \\
\hline $1.700 \mathrm{He} \mathrm{I}$ & $2.77 \pm 0.15$ & $0.28 \pm 0.03$ & $0.31 \pm 0.03$ & 0.28 \\
\hline 1.736 Br10 & $8.21 \pm 0.45$ & $0.83 \pm 0.06$ & $0.91 \pm 0.06$ & 0.90 \\
\hline $1.818 \mathrm{Br} 9$ & $14.10 \pm 0.77$ & $1.39 \pm 0.09$ & $1.52 \pm 0.10$ & 1.24 \\
\hline $1.944 \mathrm{Br} \delta$ & $13.26 \pm 0.72$ & $1.26 \pm 0.08$ & $1.38 \pm 0.09$ & 1.75 \\
\hline $1.957 \mathrm{H}_{2} 1-0 \mathrm{~S}(3)$ & $0.69 \pm 0.05$ & $0.07 \pm 0.02$ & $0.07 \pm 0.02$ & $\cdots$ \\
\hline $2.034 \mathrm{H}_{2} 1-0 \mathrm{~S}(2)$ & $0.38 \pm 0.03$ & $0.04 \pm 0.01$ & $0.04 \pm 0.01$ & $\cdots$ \\
\hline $2.058 \mathrm{He} \mathrm{I}$ & $7.27 \pm 0.40$ & $0.67 \pm 0.05$ & $0.73 \pm 0.05$ & 1.45 \\
\hline $2.073 \mathrm{H}_{2} 2-1 \mathrm{~S}(3)$ & $0.33 \pm 0.02$ & $0.03 \pm 0.01$ & $0.03 \pm 0.01$ & $\cdots$ \\
\hline $2.113 \mathrm{He} \mathrm{I}$ & $0.72 \pm 0.04$ & $0.07 \pm 0.01$ & $0.07 \pm 0.01$ & 0.08 \\
\hline $2.122 \mathrm{H}_{2} 1-0 \mathrm{~S}(1)$ & $1.13 \pm 0.06$ & $0.10 \pm 0.02$ & $0.11 \pm 0.02$ & $\cdots$ \\
\hline $2.165 \mathrm{Br} \gamma$ & $27.90 \pm 1.52$ & $2.51 \pm 0.15$ & $2.75 \pm 0.16$ & 2.61 \\
\hline $2.218[$ Fe III $]$ & $0.55 \pm 0.03$ & $0.05 \pm 0.01$ & $0.05 \pm 0.01$ & $\cdots$ \\
\hline $2.223 \mathrm{H}_{2} 1-0 \mathrm{~S}(0)$ & $0.40 \pm 0.02$ & $0.04 \pm 0.01$ & $0.05 \pm 0.01$ & $\cdots$ \\
\hline $2.248 \mathrm{H}_{2} 2-1 \mathrm{~S}(1)$ & $0.61 \pm 0.05$ & $0.06 \pm 0.02$ & $0.06 \pm 0.02$ & $\cdots$ \\
\hline $2.355 \mathrm{H}_{2} 2-1 \mathrm{~S}(0)$ & $0.22 \pm 0.02$ & $0.02 \pm 0.01$ & $0.02 \pm 0.01$ & $\cdots$ \\
\hline
\end{tabular}


Table 3-Continued

\begin{tabular}{|c|c|c|c|c|}
\hline & & & & CLOUDY \\
\hline ION & $100 \times F(\lambda) / F(\mathrm{H} \beta)^{\mathrm{a}}$ & $100 \times I(\lambda) / I(\mathrm{H} \beta)^{\mathrm{b}}$ & $100 \times I(\lambda) / I(\mathrm{H} \beta)^{\mathrm{c}}$ & stars \\
\hline
\end{tabular}

${ }^{\mathrm{a}} F(\mathrm{H} \beta)=(1.097 \pm 0.004) \times 10^{-13} \mathrm{erg} \mathrm{s}^{-1} \mathrm{~cm}^{-2}$.

${ }^{\mathrm{b}}$ Corrected for extinction with $C(\mathrm{H} \beta)=1.165$ and $\mathrm{EW}(\mathrm{abs})=0.250 \AA$.

${ }^{\mathrm{c}}$ Corrected for extinction with $C(\mathrm{H} \beta)=1.135$ and $\mathrm{EW}(\mathrm{abs})=0.100 \AA$. 
Table 4. Fluxes of optical and NIR emission lines in Mrk 71

\begin{tabular}{|c|c|c|c|c|c|}
\hline \multirow[b]{2}{*}{ ION } & \multirow[b]{2}{*}{$100 \times F(\lambda) / F(\mathrm{H} \beta)^{\mathrm{a}}$} & \multirow[b]{2}{*}{$100 \times I(\lambda) / I(\mathrm{H} \beta)^{\mathrm{b}}$} & \multirow[b]{2}{*}{$100 \times I(\lambda) / I(\mathrm{H} \beta)^{\mathrm{c}}$} & \multicolumn{2}{|c|}{ CLOUDY } \\
\hline & & & & stars & stars+shock \\
\hline \multicolumn{6}{|c|}{ a) Optical spectrum } \\
\hline $0.373[\mathrm{O}$ II $]$ & $37.61 \pm 0.56$ & $44.09 \pm 1.11$ & $44.53 \pm 1.11$ & 35.52 & 36.54 \\
\hline $0.375 \mathrm{H} 12$ & $2.58 \pm 0.11$ & $3.35 \pm 0.30$ & $3.27 \pm 0.29$ & 3.54 & 3.53 \\
\hline $0.377 \mathrm{H} 11$ & $3.02 \pm 0.11$ & $3.84 \pm 0.32$ & $3.77 \pm 0.31$ & 4.38 & 4.37 \\
\hline $0.380 \mathrm{H} 10$ & $3.98 \pm 0.11$ & $4.95 \pm 0.35$ & $4.89 \pm 0.35$ & 5.68 & 5.67 \\
\hline $0.382 \mathrm{He} \mathrm{I}$ & $0.79 \pm 0.09$ & $0.91 \pm 0.16$ & $0.92 \pm 0.16$ & 1.04 & 1.03 \\
\hline 0.384 H9 & $6.06 \pm 0.13$ & $7.35 \pm 0.43$ & $7.32 \pm 0.42$ & 7.69 & 7.67 \\
\hline $0.387[\mathrm{Ne} \mathrm{III}]$ & $52.45 \pm 0.76$ & $60.55 \pm 1.32$ & $61.27 \pm 1.32$ & 60.40 & 60.47 \\
\hline $0.389 \mathrm{He} \mathrm{I}+\mathrm{H} 8$ & $17.94 \pm 0.28$ & $21.00 \pm 0.73$ & $21.15 \pm 0.72$ & 17.52 & 17.49 \\
\hline $0.389[\mathrm{Ne} \mathrm{III}]+\mathrm{H} 7$ & $27.94 \pm 0.41$ & $32.16 \pm 0.91$ & $32.49 \pm 0.90$ & 34.51 & 34.53 \\
\hline $0.403 \mathrm{He} \mathrm{I}$ & $1.67 \pm 0.08$ & $1.89 \pm 0.21$ & $1.92 \pm 0.21$ & 1.90 & 1.89 \\
\hline 0.407 [S II] & $0.59 \pm 0.10$ & $0.67 \pm 0.14$ & $0.68 \pm 0.14$ & 0.26 & 0.32 \\
\hline $0.410 \mathrm{H} \delta$ & $24.13 \pm 0.35$ & $27.28 \pm 0.82$ & $26.80 \pm 0.80$ & 26.34 & 26.29 \\
\hline $0.434 \mathrm{H} \gamma$ & $45.40 \pm 0.65$ & $49.25 \pm 1.11$ & $48.69 \pm 1.10$ & 47.45 & 47.42 \\
\hline $0.436[\mathrm{O}$ III $]$ & $15.18 \pm 0.22$ & $16.32 \pm 0.61$ & $16.70 \pm 0.61$ & 12.63 & 12.88 \\
\hline $0.439 \mathrm{He} \mathrm{I}$ & $0.49 \pm 0.04$ & $0.52 \pm 0.11$ & $0.53 \pm 0.11$ & 0.51 & 0.51 \\
\hline $0.447 \mathrm{He} \mathrm{I}$ & $3.76 \pm 0.07$ & $3.98 \pm 0.29$ & $4.07 \pm 0.30$ & 3.95 & 3.92 \\
\hline $0.469 \mathrm{He}$ II & $0.46 \pm 0.04$ & $0.47 \pm 0.10$ & $0.49 \pm 0.10$ & 0.00 & 0.53 \\
\hline $0.471[\mathrm{Ar}$ IV $]+\mathrm{He} \mathrm{I}$ & $3.22 \pm 0.06$ & $3.28 \pm 0.26$ & $3.38 \pm 0.27$ & 2.98 & 3.01 \\
\hline $0.474[\mathrm{Ar}$ IV $]$ & $2.26 \pm 0.05$ & $2.29 \pm 0.22$ & $2.36 \pm 0.22$ & 1.83 & 1.87 \\
\hline $0.486 \mathrm{H} \beta$ & $100.00 \pm 1.42$ & $100.00 \pm 1.43$ & $100.00 \pm 1.43$ & 100.00 & 100.00 \\
\hline $0.492 \mathrm{He} \mathrm{I}$ & $0.77 \pm 0.03$ & $0.76 \pm 0.12$ & $0.79 \pm 0.13$ & 1.05 & 1.04 \\
\hline $0.496[\mathrm{O}$ III $]$ & $243.93 \pm 3.46$ & $240.18 \pm 3.56$ & $248.25 \pm 3.66$ & 229.19 & 229.53 \\
\hline 0.501 [O III $]$ & $742.38 \pm 10.5$ & $726.55 \pm 10.4$ & $751.56 \pm 10.7$ & 689.86 & 690.90 \\
\hline 0.575 [N II $]$ & $0.23 \pm 0.01$ & $0.21 \pm 0.06$ & $0.22 \pm 0.06$ & 0.02 & 0.02 \\
\hline 0.588 He I & $12.59 \pm 0.18$ & $11.33 \pm 0.46$ & $11.85 \pm 0.48$ & 10.02 & 9.94 \\
\hline $0.630[\mathrm{O} \mathrm{I}]$ & $1.05 \pm 0.02$ & $0.93 \pm 0.13$ & $0.96 \pm 0.13$ & 0.45 & 0.68 \\
\hline $0.631[\mathrm{~S}$ III $]$ & $1.55 \pm 0.02$ & $1.36 \pm 0.16$ & $1.42 \pm 0.16$ & 1.44 & 1.43 \\
\hline $0.636[\mathrm{O} \mathrm{I}]$ & $0.37 \pm 0.02$ & $0.32 \pm 0.08$ & $0.34 \pm 0.08$ & 0.15 & 0.22 \\
\hline $0.656 \mathrm{H} \alpha$ & $324.850 \pm 4.61$ & $278.68 \pm 4.41$ & $278.68 \pm 4.41$ & 281.65 & 281.90 \\
\hline 0.658 [N II] & $1.47 \pm 0.03$ & $1.26 \pm 0.15$ & $1.33 \pm 0.15$ & 0.62 & 0.66 \\
\hline $0.668 \mathrm{He} \mathrm{I}$ & $3.61 \pm 0.05$ & $3.07 \pm 0.23$ & $3.24 \pm 0.24$ & 2.70 & 2.68 \\
\hline
\end{tabular}


Table 4-Continued

\begin{tabular}{|c|c|c|c|c|c|}
\hline \multirow[b]{2}{*}{ ION } & \multirow[b]{2}{*}{$100 \times F(\lambda) / F(\mathrm{H} \beta)^{\mathrm{a}}$} & \multirow[b]{2}{*}{$100 \times I(\lambda) / I(\mathrm{H} \beta)^{\mathrm{b}}$} & \multirow[b]{2}{*}{$100 \times I(\lambda) / I(\mathrm{H} \beta)^{\mathrm{c}}$} & \multicolumn{2}{|c|}{ CLOUDY } \\
\hline & & & & stars & stars+shock \\
\hline $0.672[\mathrm{~S}$ II] & $4.14 \pm 0.06$ & $3.51 \pm 0.25$ & $3.70 \pm 0.26$ & 1.79 & 1.96 \\
\hline 0.673 [S II] & $3.43 \pm 0.05$ & $2.91 \pm 0.23$ & $3.07 \pm 0.23$ & 1.36 & 1.48 \\
\hline 0.707 He I & $4.59 \pm 0.07$ & $3.80 \pm 0.26$ & $4.02 \pm 0.27$ & 4.80 & 4.73 \\
\hline $0.714[$ Ar III $]$ & $6.54 \pm 0.10$ & $5.39 \pm 0.31$ & $5.71 \pm 0.32$ & 6.49 & 6.48 \\
\hline $0.728 \mathrm{He} \mathrm{I}$ & $0.74 \pm 0.02$ & $0.61 \pm 0.10$ & $0.64 \pm 0.11$ & 0.69 & 0.68 \\
\hline $0.732[\mathrm{O} \quad \mathrm{II}]$ & $1.05 \pm 0.02$ & $0.85 \pm 0.12$ & $0.90 \pm 0.12$ & 0.68 & 0.70 \\
\hline $0.733[\mathrm{O} \mathrm{II}]$ & $0.93 \pm 0.01$ & $0.76 \pm 0.11$ & $0.80 \pm 0.12$ & 0.54 & 0.56 \\
\hline $0.775[$ Ar III $]$ & $1.62 \pm 0.03$ & $1.28 \pm 0.15$ & $1.36 \pm 0.15$ & 1.57 & 1.57 \\
\hline $0.805[\mathrm{Cl} \mathrm{IV}]$ & $0.48 \pm 0.02$ & $0.37 \pm 0.08$ & $0.40 \pm 0.08$ & 0.38 & 0.38 \\
\hline 0.835 Pa23 & $0.21 \pm 0.02$ & $0.20 \pm 0.06$ & $0.20 \pm 0.06$ & 0.45 & 0.45 \\
\hline $0.836 \mathrm{~Pa} 22$ & $0.29 \pm 0.02$ & $0.26 \pm 0.06$ & $0.26 \pm 0.07$ & 0.48 & 0.48 \\
\hline $0.837 \mathrm{~Pa} 21$ & $0.24 \pm 0.02$ & $0.22 \pm 0.06$ & $0.22 \pm 0.06$ & 0.19 & 0.19 \\
\hline $0.839 \mathrm{~Pa} 20$ & $0.27 \pm 0.02$ & $0.23 \pm 0.06$ & $0.24 \pm 0.06$ & 0.20 & 0.20 \\
\hline 0.841 Pa19 & $0.34 \pm 0.02$ & $0.29 \pm 0.07$ & $0.30 \pm 0.07$ & 0.22 & 0.22 \\
\hline 0.844 Pa18 & $0.39 \pm 0.02$ & $0.33 \pm 0.07$ & $0.35 \pm 0.07$ & 0.25 & 0.25 \\
\hline $0.845 \mathrm{O}$ I & $0.22 \pm 0.01$ & $0.17 \pm 0.05$ & $0.18 \pm 0.05$ & 0.00 & 0.00 \\
\hline 0.847 Pa17 & $0.42 \pm 0.02$ & $0.35 \pm 0.07$ & $0.37 \pm 0.08$ & 0.29 & 0.29 \\
\hline 0.850 Pa16 & $0.66 \pm 0.02$ & $0.53 \pm 0.09$ & $0.56 \pm 0.10$ & 0.35 & 0.35 \\
\hline 0.855 Pa15 & $0.68 \pm 0.02$ & $0.54 \pm 0.09$ & $0.57 \pm 0.10$ & 0.45 & 0.45 \\
\hline $0.860 \mathrm{~Pa} 14$ & $0.83 \pm 0.02$ & $0.65 \pm 0.10$ & $0.69 \pm 0.11$ & 0.98 & 0.98 \\
\hline 0.867 Pa13 & $0.99 \pm 0.02$ & $0.78 \pm 0.11$ & $0.82 \pm 0.12$ & 1.02 & 1.02 \\
\hline 0.875 Pa12 & $1.33 \pm 0.03$ & $1.02 \pm 0.13$ & $1.08 \pm 0.13$ & 1.19 & 1.19 \\
\hline 0.886 Pa11 & $1.78 \pm 0.03$ & $1.35 \pm 0.14$ & $1.43 \pm 0.15$ & 1.48 & 1.48 \\
\hline $0.901 \mathrm{~Pa} 10$ & $1.57 \pm 0.03$ & $1.19 \pm 0.14$ & $1.27 \pm 0.14$ & 1.91 & 1.91 \\
\hline 0.907 [S III $]$ & $11.63 \pm 0.17$ & $8.59 \pm 0.38$ & $9.22 \pm 0.40$ & 10.46 & 10.47 \\
\hline 0.923 Pa9 & $3.17 \pm 0.06$ & $2.34 \pm 0.19$ & $2.51 \pm 0.20$ & 2.57 & 2.56 \\
\hline 0.953 [S III] & $31.61 \pm 0.45$ & $22.91 \pm 0.67$ & $24.64 \pm 0.71$ & 25.94 & 25.95 \\
\hline $0.955 \mathrm{~Pa} \epsilon$ & $5.06 \pm 0.07$ & $3.68 \pm 0.24$ & $3.96 \pm 0.25$ & 3.63 & 3.62 \\
\hline & b) Near & infrared spectrum & & & \\
\hline 0.907 [S III] & $15.88 \pm 1.46$ & $11.69 \pm 1.09$ & $12.56 \pm 1.17$ & 10.46 & 10.47 \\
\hline 0.953 [S III] & $31.61 \pm 1.72$ & $22.91 \pm 1.30$ & $24.64 \pm 1.40$ & 25.94 & 25.95 \\
\hline $0.955 \mathrm{~Pa} \epsilon$ & $4.68 \pm 0.27$ & $3.42 \pm 0.26$ & $3.67 \pm 0.27$ & 3.63 & 3.62 \\
\hline
\end{tabular}


Table 4-Continued

\begin{tabular}{|c|c|c|c|c|c|}
\hline \multirow[b]{2}{*}{ ION } & \multirow[b]{2}{*}{$100 \times F(\lambda) / F(\mathrm{H} \beta)^{\mathrm{a}}$} & \multirow[b]{2}{*}{$100 \times I(\lambda) / I(\mathrm{H} \beta)^{\mathrm{b}}$} & \multirow[b]{2}{*}{$100 \times I(\lambda) / I(\mathrm{H} \beta)^{\mathrm{c}}$} & \multicolumn{2}{|c|}{ CLOUDY } \\
\hline & & & & stars & stars+shock \\
\hline $1.003 \mathrm{He} \mathrm{I}$ & $0.23 \pm 0.02$ & $0.17 \pm 0.05$ & $0.18 \pm 0.05$ & 0.12 & 0.12 \\
\hline $1.005 \mathrm{~Pa} \delta$ & $7.05 \pm 0.39$ & $5.04 \pm 0.33$ & $5.44 \pm 0.36$ & 5.42 & 5.41 \\
\hline $1.032[\mathrm{~S} \mathrm{II}]$ & $0.11 \pm 0.02$ & $0.08 \pm 0.03$ & $0.08 \pm 0.04$ & 0.08 & 0.09 \\
\hline $1.083 \mathrm{He} \mathrm{I}$ & $45.34 \pm 2.46$ & $31.64 \pm 1.79$ & $34.24 \pm 1.94$ & 27.71 & 27.53 \\
\hline $1.091 \mathrm{He} \mathrm{I}$ & $0.51 \pm 0.03$ & $0.36 \pm 0.07$ & $0.39 \pm 0.08$ & 0.28 & 0.28 \\
\hline $1.094 \mathrm{~Pa} \gamma$ & $12.47 \pm 0.68$ & $8.70 \pm 0.52$ & $9.41 \pm 0.56$ & 8.69 & 8.67 \\
\hline $1.162 \mathrm{H}_{2} 2-0 \mathrm{~S}(1)$ & $0.03 \pm 0.01$ & $0.02 \pm 0.01$ & $0.02 \pm 0.01$ & $\cdots$ & $\cdots$ \\
\hline $1.197 \mathrm{He} \mathrm{I}$ & $0.29 \pm 0.02$ & $0.20 \pm 0.05$ & $0.21 \pm 0.06$ & 0.19 & 0.19 \\
\hline $1.207 \mathrm{H}_{2} 3-1 \mathrm{~S}(2)$ & $0.06 \pm 0.01$ & $0.04 \pm 0.02$ & $0.04 \pm 0.02$ & $\cdots$ & $\cdots$ \\
\hline $1.233 \mathrm{H}_{2} 3-1 \mathrm{~S}(1)$ & $0.12 \pm 0.01$ & $0.08 \pm 0.03$ & $0.09 \pm 0.03$ & $\cdots$ & $\cdots$ \\
\hline $1.238 \mathrm{H}_{2} 2-0 \mathrm{Q}(1)$ & $0.11 \pm 0.01$ & $0.07 \pm 0.03$ & $0.08 \pm 0.03$ & $\cdots$ & $\cdots$ \\
\hline $1.253 \mathrm{He} \mathrm{I}$ & $0.21 \pm 0.02$ & $0.14 \pm 0.05$ & $0.16 \pm 0.05$ & 0.28 & 0.28 \\
\hline $1.257[\mathrm{Fe} \mathrm{II}]$ & $0.12 \pm 0.01$ & $0.08 \pm 0.03$ & $0.09 \pm 0.04$ & 0.28 & 0.41 \\
\hline $1.279 \mathrm{He} \mathrm{I}$ & $1.19 \pm 0.07$ & $0.80 \pm 0.11$ & $0.87 \pm 0.11$ & 0.69 & 0.68 \\
\hline $1.282 \mathrm{~Pa} \beta$ & $21.73 \pm 1.18$ & $14.61 \pm 0.85$ & $15.88 \pm 0.92$ & 15.45 & 15.43 \\
\hline $1.311 \mathrm{H}_{2} 4-2 \mathrm{~S}(1)$ & $0.11 \pm 0.01$ & $0.07 \pm 0.03$ & $0.08 \pm 0.03$ & $\cdots$ & $\cdots$ \\
\hline $1.314 \mathrm{H}_{2} 3-1 \mathrm{Q}(1)$ & $0.11 \pm 0.01$ & $0.07 \pm 0.03$ & $0.08 \pm 0.03$ & $\cdots$ & $\cdots$ \\
\hline $1.514 \mathrm{Br} 21$ & $0.15 \pm 0.01$ & $0.10 \pm 0.04$ & $0.11 \pm 0.04$ & 0.09 & 0.09 \\
\hline 1.519 Br20 & $0.17 \pm 0.01$ & $0.12 \pm 0.04$ & $0.13 \pm 0.04$ & 0.10 & 0.10 \\
\hline 1.526 Br19 & $0.25 \pm 0.02$ & $0.17 \pm 0.05$ & $0.18 \pm 0.05$ & 0.10 & 0.10 \\
\hline 1.535 $\mathrm{Br} 18$ & $0.21 \pm 0.01$ & $0.14 \pm 0.04$ & $0.16 \pm 0.05$ & 0.12 & 0.12 \\
\hline 1.544 Br17 & $0.24 \pm 0.02$ & $0.17 \pm 0.05$ & $0.18 \pm 0.05$ & 0.14 & 0.14 \\
\hline 1.556 Br16 & $0.37 \pm 0.02$ & $0.25 \pm 0.06$ & $0.27 \pm 0.06$ & 0.17 & 0.17 \\
\hline 1.570 $\mathrm{Br} 15$ & $0.46 \pm 0.03$ & $0.31 \pm 0.06$ & $0.33 \pm 0.07$ & 0.22 & 0.22 \\
\hline $1.588 \mathrm{Br} 14$ & $0.44 \pm 0.03$ & $0.29 \pm 0.06$ & $0.32 \pm 0.07$ & 0.47 & 0.47 \\
\hline $1.601 \mathrm{H}_{2} 6-4 \mathrm{Q}(1)$ & $0.03 \pm 0.01$ & $0.02 \pm 0.01$ & $0.02 \pm 0.01$ & $\cdots$ & $\cdots$ \\
\hline $1.611 \mathrm{Br} 13$ & $0.61 \pm 0.03$ & $0.40 \pm 0.07$ & $0.43 \pm 0.08$ & 0.49 & 0.49 \\
\hline 1.641 $\mathrm{Br} 12$ & $0.69 \pm 0.04$ & $0.45 \pm 0.08$ & $0.49 \pm 0.08$ & 0.57 & 0.57 \\
\hline 1.681 Br11 & $0.95 \pm 0.05$ & $0.61 \pm 0.09$ & $0.67 \pm 0.10$ & 0.71 & 0.71 \\
\hline $1.700 \mathrm{He} \mathrm{I}$ & $0.22 \pm 0.01$ & $0.14 \pm 0.04$ & $0.16 \pm 0.05$ & 0.28 & 0.28 \\
\hline 1.736 $\mathrm{Br} 10$ & $1.32 \pm 0.07$ & $0.85 \pm 0.11$ & $0.93 \pm 0.12$ & 0.91 & 0.91 \\
\hline $1.748 \mathrm{He} \mathrm{I}$ & $0.10 \pm 0.01$ & $0.06 \pm 0.03$ & $0.07 \pm 0.03$ & 0.00 & 0.00 \\
\hline
\end{tabular}


Table 4-Continued

\begin{tabular}{lrrrrc}
\hline \hline & & & & \multicolumn{2}{c}{ CLOUDY } \\
\cline { 5 - 6 } ION & $100 \times F(\lambda) / F(\mathrm{H} \beta)^{\mathrm{a}}$ & $100 \times I(\lambda) / I(\mathrm{H} \beta)^{\mathrm{b}}$ & $100 \times I(\lambda) / I(\mathrm{H} \beta)^{\mathrm{c}}$ & stars & stars+shock \\
\hline $1.818 \mathrm{Br} 9$ & $2.19 \pm 0.12$ & $1.40 \pm 0.14$ & $1.54 \pm 0.15$ & 1.23 & 1.23 \\
$1.944 \mathrm{Br} \delta$ & $2.72 \pm 0.15$ & $1.72 \pm 0.16$ & $1.89 \pm 0.17$ & 1.74 & 1.73 \\
$1.957 \mathrm{H}_{2} 1-0 \mathrm{~S}(3)$ & $0.30 \pm 0.03$ & $0.19 \pm 0.05$ & $0.21 \pm 0.05$ & $\ldots$ & $\ldots$ \\
$2.034 \mathrm{H}_{2} 1-0 \mathrm{~S}(2)$ & $0.07 \pm 0.01$ & $0.05 \pm 0.02$ & $0.05 \pm 0.03$ & $\ldots$ & $\ldots$ \\
$2.058 \mathrm{He}$ & $1.39 \pm 0.08$ & $0.88 \pm 0.11$ & $0.96 \pm 0.12$ & 1.45 & 1.44 \\
$2.073 \mathrm{H}_{2} 2-1 \mathrm{~S}(3)$ & $0.07 \pm 0.01$ & $0.05 \pm 0.02$ & $0.05 \pm 0.03$ & $\ldots$ & $\ldots$ \\
$2.122 \mathrm{H}_{2} 1-0 \mathrm{~S}(1)$ & $0.33 \pm 0.02$ & $0.21 \pm 0.05$ & $0.23 \pm 0.06$ & $\ldots$ & $\ldots$ \\
$2.165 \mathrm{Br}$ & $3.51 \pm 0.19$ & $2.20 \pm 0.18$ & $2.42 \pm 0.20$ & 2.59 & 2.58 \\
$2.223 \mathrm{H}_{2} 1-0 \mathrm{~S}(0)$ & $0.16 \pm 0.01$ & $0.10 \pm 0.04$ & $0.11 \pm 0.04$ & $\ldots$ & $\ldots$ \\
$2.248 \mathrm{H}_{2} 2-1 \mathrm{~S}(1)$ & $0.15 \pm 0.01$ & $0.10 \pm 0.04$ & $0.11 \pm 0.04$ & $\ldots$ & $\ldots$ \\
$2.355 \mathrm{H}_{2} 2-1 \mathrm{~S}(0)$ & $0.08 \pm 0.01$ & $0.05 \pm 0.03$ & $0.05 \pm 0.03$ & $\ldots$ & $\ldots$ \\
\hline
\end{tabular}

${ }^{\mathrm{a}} F(\mathrm{H} \beta)=(4.112 \pm 0.004) \times 10^{-13} \mathrm{erg} \mathrm{s}^{-1} \mathrm{~cm}^{-2}$.

${ }^{\mathrm{b}}$ Corrected for extinction with $C(\mathrm{H} \beta)=0.225$ and $\mathrm{EW}(\mathrm{abs})=0.6 \AA$.

${ }^{\mathrm{c}}$ Corrected for extinction with $C(\mathrm{H} \beta)=0.195$ and $\mathrm{EW}(\mathrm{abs})=0.4 \AA$. 
Table 5. Fluxes of optical and NIR emission lines in Mrk 930

\begin{tabular}{|c|c|c|c|c|c|}
\hline \multirow[b]{2}{*}{ ION } & \multirow[b]{2}{*}{$100 \times F(\lambda) / F(\mathrm{H} \beta)^{\mathrm{a}}$} & \multirow[b]{2}{*}{$100 \times I(\lambda) / I(\mathrm{H} \beta)^{\mathrm{b}}$} & \multirow[b]{2}{*}{$100 \times I(\lambda) / I(\mathrm{H} \beta)^{\mathrm{c}}$} & \multicolumn{2}{|c|}{ CLOUDY } \\
\hline & & & & stars & stars+shock \\
\hline \multicolumn{6}{|c|}{ a) Optical spectrum } \\
\hline $0.373[\mathrm{O}$ II $]$ & $207.01 \pm 3.37$ & $250.96 \pm 4.94$ & $249.57 \pm 4.97$ & 259.92 & 261.91 \\
\hline 0.384 H9 & $3.66 \pm 0.31$ & $9.16 \pm 0.92$ & $10.30 \pm 1.04$ & 7.70 & 7.68 \\
\hline 0.387 [Ne III $]$ & $29.83 \pm 0.70$ & $35.40 \pm 1.29$ & $35.30 \pm 1.30$ & 38.61 & 39.17 \\
\hline $0.389 \mathrm{He} \mathrm{I}+\mathrm{H} 8$ & $12.66 \pm 0.46$ & $20.00 \pm 1.03$ & $21.19 \pm 1.07$ & 21.48 & 21.40 \\
\hline $0.389[\mathrm{Ne}$ III $]+\mathrm{H} 7$ & $20.54 \pm 0.58$ & $28.98 \pm 1.19$ & $30.20 \pm 1.23$ & 27.94 & 28.12 \\
\hline $0.407[\mathrm{~S}$ II $]$ & $1.92 \pm 0.46$ & $2.20 \pm 0.56$ & $2.21 \pm 0.56$ & 1.45 & 1.63 \\
\hline $0.410 \mathrm{H} \delta$ & $19.58 \pm 0.55$ & $26.82 \pm 1.12$ & $27.19 \pm 1.14$ & 26.29 & 26.24 \\
\hline $0.434 \mathrm{H} \gamma$ & $39.27 \pm 0.82$ & $46.33 \pm 1.42$ & $46.28 \pm 1.44$ & 47.32 & 47.29 \\
\hline $0.436[\mathrm{O}$ III $]$ & $4.84 \pm 0.38$ & $5.21 \pm 0.51$ & $5.26 \pm 0.52$ & 5.71 & 5.84 \\
\hline 0.447 He I & $3.38 \pm 0.38$ & $3.55 \pm 0.46$ & $3.60 \pm 0.47$ & 4.14 & 4.12 \\
\hline $0.466[\mathrm{Fe} \quad \mathrm{III}]$ & $1.71 \pm 0.35$ & $1.73 \pm 0.38$ & $1.76 \pm 0.39$ & 1.69 & 1.69 \\
\hline 0.469 He II & $0.83 \pm 0.23$ & $0.84 \pm 0.25$ & $0.85 \pm 0.25$ & 0.00 & 0.80 \\
\hline $0.486 \mathrm{H} \beta$ & $100.00 \pm 1.76$ & $100.00 \pm 1.82$ & $100.00 \pm 1.85$ & 100.00 & 100.00 \\
\hline 0.496 [O III $]$ & $147.18 \pm 2.50$ & $140.29 \pm 2.75$ & $143.91 \pm 2.85$ & 148.71 & 148.74 \\
\hline $0.501[\mathrm{O}$ III $]$ & $445.01 \pm 7.23$ & $420.63 \pm 7.18$ & $431.96 \pm 7.47$ & 447.61 & 447.70 \\
\hline $0.588 \mathrm{He} \mathrm{I}$ & $13.09 \pm 0.43$ & $11.02 \pm 0.59$ & $11.49 \pm 0.62$ & 10.26 & 10.21 \\
\hline $0.630[\mathrm{O}$ I $]$ & $7.67 \pm 0.41$ & $6.19 \pm 0.46$ & $6.49 \pm 0.48$ & 3.58 & 5.18 \\
\hline $0.631[\mathrm{~S} \mathrm{III}]$ & $1.64 \pm 0.48$ & $1.32 \pm 0.40$ & $1.39 \pm 0.42$ & 1.61 & 1.61 \\
\hline $0.636[\mathrm{O} \mathrm{I}]$ & $2.12 \pm 0.26$ & $1.70 \pm 0.26$ & $1.78 \pm 0.27$ & 1.14 & 1.65 \\
\hline $0.656 \mathrm{H} \alpha$ & $358.66 \pm 5.86$ & $284.11 \pm 5.33$ & $284.18 \pm 5.43$ & 285.23 & 285.75 \\
\hline $0.658[\mathrm{~N} \mathrm{II}]$ & $18.19 \pm 0.51$ & $14.30 \pm 0.66$ & $15.04 \pm 0.69$ & 9.51 & 9.78 \\
\hline 0.668 He I & $4.02 \pm 0.30$ & $3.13 \pm 0.32$ & $3.30 \pm 0.34$ & 2.90 & 2.88 \\
\hline $0.672[\mathrm{~S}$ II $]$ & $34.74 \pm 0.75$ & $26.97 \pm 0.92$ & $28.41 \pm 0.97$ & 9.47 & 10.31 \\
\hline $0.673[\mathrm{~S} \mathrm{II}]$ & $25.53 \pm 0.65$ & $19.79 \pm 0.78$ & $20.85 \pm 0.83$ & 6.71 & 7.30 \\
\hline $0.707 \mathrm{He} \mathrm{I}$ & $2.46 \pm 0.22$ & $1.85 \pm 0.24$ & $1.95 \pm 0.25$ & 2.21 & 2.20 \\
\hline 0.714 [Ar III $]$ & $9.27 \pm 0.34$ & $6.92 \pm 0.44$ & $7.33 \pm 0.47$ & 6.93 & 6.92 \\
\hline \multicolumn{6}{|c|}{ b) Near infrared spectrum } \\
\hline $0.953[\mathrm{~S}$ III $]$ & $61.39 \pm 1.17$ & $38.30 \pm 1.16$ & $36.77 \pm 1.12$ & 38.48 & 38.60 \\
\hline $0.955 \mathrm{~Pa} \epsilon$ & $4.68 \pm 0.32$ & $3.77 \pm 0.34$ & $3.82 \pm 0.34$ & 3.68 & 3.67 \\
\hline $1.005 \mathrm{~Pa} \delta$ & $7.78 \pm 0.26$ & $5.81 \pm 0.37$ & $5.84 \pm 0.37$ & 5.50 & 5.49 \\
\hline $1.029[\mathrm{~S} \mathrm{II}]$ & $1.71 \pm 0.15$ & $2.00 \pm 0.25$ & $2.15 \pm 0.27$ & 0.25 & 0.25 \\
\hline
\end{tabular}


Table 5-Continued

\begin{tabular}{lrrrrr}
\hline \hline & & & & \multicolumn{2}{c}{ CLOUDY } \\
\cline { 5 - 6 } \multicolumn{1}{c}{ ION } & $100 \times F(\lambda) / F(\mathrm{H} \beta)^{\mathrm{a}}$ & $100 \times I(\lambda) / I(\mathrm{H} \beta)^{\mathrm{b}}$ & $100 \times I(\lambda) / I(\mathrm{H} \beta)^{\mathrm{c}}$ & stars & stars+shock \\
\hline & $36.44 \pm 0.65$ & $22.43 \pm 0.80$ & $21.87 \pm 0.78$ & 20.00 & 19.90 \\
$1.083 \mathrm{He} \mathrm{I}$ & $14.16 \pm 0.30$ & $9.21 \pm 0.46$ & $9.10 \pm 0.46$ & 8.84 & 8.82 \\
$1.094 \mathrm{~Pa} \gamma$ & $3.42 \pm 0.19$ & $2.23 \pm 0.31$ & $2.19 \pm 0.30$ & 1.30 & 1.74 \\
$1.257[\mathrm{Fe} \mathrm{II}]$ & $27.07 \pm 0.47$ & $15.81 \pm 0.64$ & $15.51 \pm 0.63$ & 15.81 & 15.79 \\
$1.282 \mathrm{~Pa} \beta$ & $3.70 \pm 0.21$ & $1.97 \pm 0.20$ & $1.93 \pm 0.20$ & 1.26 & 1.69 \\
$1.643[\mathrm{Fe} \mathrm{II}]$ & $2.14 \pm 0.12$ & $1.45 \pm 0.17$ & $1.50 \pm 0.17$ & 0.93 & 0.93 \\
$1.736 \mathrm{Br} 10$ & $69.09 \pm 3.63$ & $36.36 \pm 2.14$ & $35.82 \pm 2.12$ & 32.28 & 32.25 \\
$1.876 \mathrm{~Pa} \alpha$ & $3.70 \pm 0.20$ & $2.14 \pm 0.21$ & $2.15 \pm 0.21$ & 1.79 & 1.79 \\
$1.944 \mathrm{Br} \delta$ & $1.73 \pm 0.09$ & $1.09 \pm 0.14$ & $1.12 \pm 0.14$ & 1.48 & 1.47 \\
$2.058 \mathrm{He} \mathrm{I}$ & $1.13 \pm 0.06$ & $0.76 \pm 0.12$ & $0.79 \pm 0.12$ & $\ldots$ & $\ldots$ \\
$2.122 \mathrm{H} \mathrm{H}_{2} 1-0 \mathrm{~S}(1)$ & $4.59 \pm 0.24$ & $2.50 \pm 0.22$ & $2.50 \pm 0.23$ & 2.68 & 2.67 \\
$2.165 \mathrm{Br} \gamma$ & $0.85 \pm 0.05$ & $0.46 \pm 0.09$ & $0.43 \pm 0.09$ & $\ldots$ & $\ldots$ \\
$2.223 \mathrm{H}_{2} 1-0 \mathrm{~S}(1)$ & $0.65 \pm 0.04$ & $0.35 \pm 0.08$ & $0.32 \pm 0.08$ & $\ldots$ & $\ldots$ \\
$2.248 \mathrm{H}_{2} 2-1 \mathrm{~S}(1)$ & & & & & $\ldots$
\end{tabular}

${ }^{\mathrm{a}} F(\mathrm{H} \beta)=(6.937 \pm 0.051) \times 10^{-14} \mathrm{erg} \mathrm{s}^{-1} \mathrm{~cm}^{-2}$.

${ }^{\mathrm{b}}$ Corrected for extinction with $C(\mathrm{H} \beta)=0.310$ and $\mathrm{EW}(\mathrm{abs})=2.85 \AA$.

${ }^{\mathrm{c}}$ Corrected for extinction with $C(\mathrm{H} \beta)=0.270$ and $\mathrm{EW}(\mathrm{abs})=3.55 \AA$. 
Table 6. Fluxes of optical and NIR emission lines in Mrk 996

\begin{tabular}{|c|c|c|c|c|}
\hline ION & $100 \times F(\lambda) / F(\mathrm{H} \beta)^{\mathrm{a}}$ & $100 \times I(\lambda) / I(\mathrm{H} \beta)^{\mathrm{b}}$ & $100 \times I(\lambda) / I(\mathrm{H} \beta)^{\mathrm{c}}$ & $\frac{\text { CLOUDY }}{\text { stars }}$ \\
\hline \multicolumn{5}{|c|}{ a) Optical spectrum } \\
\hline $0.373[\mathrm{O}$ II] & $149.06 \pm 2.33$ & $198.11 \pm 3.78$ & $199.88 \pm 3.80$ & 157.30 \\
\hline 0.377 H11 & $0.83 \pm 0.29$ & $6.01 \pm 2.77$ & $6.07 \pm 2.80$ & 4.64 \\
\hline $0.380 \mathrm{H} 10$ & $1.54 \pm 0.34$ & $7.14 \pm 2.04$ & $7.21 \pm 2.06$ & 5.95 \\
\hline 0.384 H9 & $2.59 \pm 0.28$ & $8.37 \pm 1.21$ & $8.46 \pm 1.22$ & 7.98 \\
\hline 0.387 [Ne III $]$ & $38.04 \pm 0.73$ & $49.01 \pm 1.41$ & $49.55 \pm 1.41$ & 44.15 \\
\hline $0.389 \mathrm{He} \mathrm{I}+\mathrm{H} 8$ & $11.82 \pm 0.38$ & $20.60 \pm 0.98$ & $20.84 \pm 0.99$ & 19.70 \\
\hline $0.389[\mathrm{Ne} \mathrm{III}]+\mathrm{H} 7$ & $18.47 \pm 0.45$ & $28.33 \pm 1.08$ & $28.69 \pm 1.08$ & 29.87 \\
\hline 0.403 He I & $2.24 \pm 0.26$ & $2.77 \pm 0.38$ & $2.80 \pm 0.38$ & 2.79 \\
\hline $0.407[\mathrm{~S} \mathrm{II}]$ & $1.12 \pm 0.16$ & $1.37 \pm 0.24$ & $1.39 \pm 0.24$ & 2.16 \\
\hline $0.410 \mathrm{H} \delta$ & $20.60 \pm 0.45$ & $29.95 \pm 1.07$ & $29.49 \pm 1.06$ & 26.99 \\
\hline $0.434 \mathrm{H} \gamma$ & $45.21 \pm 0.73$ & $55.46 \pm 1.39$ & $54.88 \pm 1.38$ & 47.94 \\
\hline $0.436[\mathrm{O}$ III $]$ & $13.27 \pm 0.34$ & $14.83 \pm 0.68$ & $15.13 \pm 0.68$ & 17.46 \\
\hline 0.447 He I & $6.35 \pm 0.23$ & $6.86 \pm 0.45$ & $7.02 \pm 0.46$ & 6.10 \\
\hline $0.466[\mathrm{Fe} \mathrm{III}]$ & $2.40 \pm 0.19$ & $2.45 \pm 0.28$ & $2.51 \pm 0.28$ & 2.36 \\
\hline $0.486 \mathrm{H} \beta$ & $100.00 \pm 1.49$ & $100.00 \pm 1.57$ & $100.00 \pm 1.58$ & 100.00 \\
\hline $0.492 \mathrm{He} \mathrm{I}$ & $1.07 \pm 0.17$ & $1.01 \pm 0.20$ & $1.04 \pm 0.20$ & 1.52 \\
\hline $0.496[\mathrm{O}$ III $]$ & $98.73 \pm 1.47$ & $92.55 \pm 1.74$ & $95.58 \pm 1.79$ & 79.20 \\
\hline $0.501[\mathrm{O} \mathrm{III}]$ & $304.25 \pm 4.44$ & $281.75 \pm 4.42$ & $291.21 \pm 4.57$ & 238.40 \\
\hline $0.520[\mathrm{~N} \mathrm{I}]$ & $0.62 \pm 0.11$ & $0.55 \pm 0.13$ & $0.57 \pm 0.13$ & 0.33 \\
\hline $0.588 \mathrm{He} \mathrm{I}$ & $19.00 \pm 0.32$ & $14.88 \pm 0.56$ & $15.55 \pm 0.58$ & 18.62 \\
\hline 0.630 [O I] & $4.40 \pm 0.12$ & $3.24 \pm 0.25$ & $3.40 \pm 0.26$ & 1.62 \\
\hline 0.631 [S III] & $2.46 \pm 0.11$ & $1.81 \pm 0.19$ & $1.90 \pm 0.19$ & 3.89 \\
\hline $0.656 \mathrm{H} \alpha$ & $377.58 \pm 5.49$ & $270.24 \pm 4.53$ & $270.07 \pm 4.54$ & 280.93 \\
\hline $0.658[\mathrm{~N} \mathrm{II}]$ & $26.68 \pm 0.41$ & $18.91 \pm 0.61$ & $19.89 \pm 0.64$ & 25.85 \\
\hline 0.668 He I & $5.78 \pm 0.14$ & $4.04 \pm 0.27$ & $4.26 \pm 0.28$ & 4.15 \\
\hline $0.672[\mathrm{~S}$ II $]$ & $22.06 \pm 0.34$ & $15.35 \pm 0.54$ & $16.17 \pm 0.56$ & 14.39 \\
\hline $0.673[\mathrm{~S}$ II $]$ & $17.44 \pm 0.28$ & $12.11 \pm 0.47$ & $12.76 \pm 0.50$ & 11.22 \\
\hline $0.707 \mathrm{He} \mathrm{I}$ & $10.71 \pm 0.19$ & $7.11 \pm 0.36$ & $7.51 \pm 0.37$ & 13.34 \\
\hline 0.714 [Ar III] & $10.86 \pm 0.19$ & $7.14 \pm 0.35$ & $7.55 \pm 0.37$ & 6.69 \\
\hline $0.732[\mathrm{O}$ II $]$ & $4.27 \pm 0.10$ & $2.74 \pm 0.21$ & $2.90 \pm 0.22$ & 5.36 \\
\hline $0.733[\mathrm{O} \quad \mathrm{II}]$ & $2.79 \pm 0.09$ & $1.78 \pm 0.17$ & $1.89 \pm 0.18$ & 4.26 \\
\hline
\end{tabular}


Table 6-Continued

\begin{tabular}{|c|c|c|c|c|}
\hline ION & $100 \times F(\lambda) / F(\mathrm{H} \beta)^{\mathrm{a}}$ & $100 \times I(\lambda) / I(\mathrm{H} \beta)^{\mathrm{b}}$ & $100 \times I(\lambda) / I(\mathrm{H} \beta)^{\mathrm{c}}$ & $\frac{\text { CLOUDY }}{\text { stars }}$ \\
\hline $0.775[$ Ar III $]$ & $2.32 \pm 0.09$ & $1.40 \pm 0.15$ & $1.49 \pm 0.16$ & 1.61 \\
\hline 0.907 [S III $]$ & $23.82 \pm 0.40$ & $13.41 \pm 0.47$ & $14.40 \pm 0.50$ & 14.40 \\
\hline $0.953[\mathrm{~S} \mathrm{III}]$ & $70.07 \pm 1.07$ & $35.50 \pm 0.90$ & $38.20 \pm 0.97$ & 35.71 \\
\hline \multicolumn{5}{|c|}{ b) Near infrared spectrum } \\
\hline 0.953 [S III] & $70.07 \pm 3.91$ & $35.50 \pm 2.12$ & $38.20 \pm 2.29$ & 35.71 \\
\hline $0.955 \mathrm{~Pa} \epsilon$ & $3.85 \pm 0.40$ & $2.56 \pm 0.31$ & $2.76 \pm 0.33$ & 3.67 \\
\hline $1.005 \mathrm{~Pa} \delta$ & $9.29 \pm 0.54$ & $5.17 \pm 0.36$ & $5.58 \pm 0.38$ & 5.46 \\
\hline $1.083 \mathrm{He} \mathrm{I}$ & $132.86 \pm 7.32$ & $62.56 \pm 3.71$ & $67.64 \pm 4.01$ & 218.83 \\
\hline $1.094 \mathrm{~Pa} \gamma$ & $15.32 \pm 0.85$ & $7.70 \pm 0.49$ & $8.33 \pm 0.52$ & 8.82 \\
\hline $1.257[\mathrm{Fe} \mathrm{II}]$ & $2.30 \pm 0.15$ & $1.01 \pm 0.11$ & $1.10 \pm 0.12$ & 2.53 \\
\hline $1.279 \mathrm{He} \mathrm{I}$ & $2.12 \pm 0.18$ & $0.93 \pm 0.11$ & $1.01 \pm 0.12$ & 0.53 \\
\hline $1.282 \mathrm{~Pa} \beta$ & $30.25 \pm 1.67$ & $13.60 \pm 0.83$ & $14.78 \pm 0.90$ & 15.54 \\
\hline $1.643[\mathrm{Fe} \mathrm{II}]$ & $2.28 \pm 0.13$ & $0.92 \pm 0.10$ & $1.00 \pm 0.11$ & 2.44 \\
\hline 1.736 Br10 & $2.50 \pm 0.16$ & $1.23 \pm 0.12$ & $1.35 \pm 0.13$ & 0.92 \\
\hline $1.944 \mathrm{Br} \delta^{\mathrm{c}}$ & $4.82 \pm 0.38$ & $2.07 \pm 0.19$ & $2.27 \pm 0.21$ & 1.74 \\
\hline $1.957 \mathrm{H}_{2} 1-0 \mathrm{~S}(3)$ & $0.36 \pm 0.03$ & $0.15 \pm 0.05$ & $0.16 \pm 0.05$ & $\ldots$ \\
\hline $2.034 \mathrm{H}_{2} 1-0 \mathrm{~S}(2)$ & $0.28 \pm 0.03$ & $0.11 \pm 0.04$ & $0.12 \pm 0.04$ & $\cdots$ \\
\hline $2.122 \mathrm{H}_{2} 1-0 \mathrm{~S}(1)$ & $1.06 \pm 0.06$ & $0.40 \pm 0.06$ & $0.44 \pm 0.07$ & $\cdots$ \\
\hline $2.165 \mathrm{Br} \gamma$ & $6.07 \pm 0.34$ & $2.46 \pm 0.18$ & $2.70 \pm 0.20$ & 2.60 \\
\hline $2.248 \mathrm{H}_{2} 2-1 \mathrm{~S}(1)$ & $0.34 \pm 0.03$ & $0.13 \pm 0.04$ & $0.14 \pm 0.04$ & $\ldots$ \\
\hline $2.355 \mathrm{H}_{2} 2-1 \mathrm{~S}(0)$ & $0.21 \pm 0.03$ & $0.08 \pm 0.03$ & $0.08 \pm 0.03$ & $\cdots$ \\
\hline
\end{tabular}

${ }^{\mathrm{a}} F(\mathrm{H} \beta)=(7.241 \pm 0.023) \times 10^{-14} \mathrm{erg} \mathrm{s}^{-1} \mathrm{~cm}^{-2}$.

${ }^{\mathrm{b}}$ Corrected for extinction with $C(\mathrm{H} \beta)=0.450$ and $\mathrm{EW}(\mathrm{abs})=2 \AA$.

${ }^{\mathrm{c}}$ Corrected for extinction with $C(\mathrm{H} \beta)=0.420$ and $\mathrm{EW}(\mathrm{abs})=2 \AA$. 
Table 7. Fluxes of optical and NIR emission lines in SBS 0335-052E

\begin{tabular}{|c|c|c|c|c|c|}
\hline \multirow[b]{2}{*}{ ION } & \multirow[b]{2}{*}{$100 \times F(\lambda) / F(\mathrm{H} \beta)^{\mathrm{a}}$} & \multirow[b]{2}{*}{$100 \times I(\lambda) / I(\mathrm{H} \beta)^{\mathrm{b}}$} & \multirow[b]{2}{*}{$100 \times I(\lambda) / I(\mathrm{H} \beta)^{\mathrm{c}}$} & \multicolumn{2}{|c|}{ CLOUDY } \\
\hline & & & & stars & stars+shock \\
\hline \multicolumn{6}{|c|}{ a) Optical spectrum } \\
\hline $0.373[\mathrm{O} \mathrm{II}]$ & $28.34 \pm 0.47$ & $31.11 \pm 0.91$ & $31.43 \pm 0.90$ & 27.36 & 27.17 \\
\hline $0.375 \mathrm{H} 12$ & $2.25 \pm 0.18$ & $2.94 \pm 0.39$ & $2.83 \pm 0.39$ & 3.43 & 3.38 \\
\hline $0.377 \mathrm{H} 11$ & $3.85 \pm 0.16$ & $5.10 \pm 0.60$ & $4.89 \pm 0.60$ & 4.26 & 4.20 \\
\hline $0.380 \mathrm{H} 10$ & $4.99 \pm 0.17$ & $6.15 \pm 0.52$ & $6.01 \pm 0.52$ & 5.52 & 5.44 \\
\hline $0.384 \mathrm{H} 9$ & $7.61 \pm 0.18$ & $8.98 \pm 0.56$ & $8.88 \pm 0.56$ & 7.49 & 7.38 \\
\hline 0.387 [Ne III $]$ & $22.27 \pm 0.36$ & $24.23 \pm 0.78$ & $24.52 \pm 0.78$ & 24.66 & 23.73 \\
\hline $0.389 \mathrm{He} \mathrm{I}+\mathrm{H} 8$ & $16.03 \pm 0.28$ & $17.94 \pm 0.69$ & $18.00 \pm 0.68$ & 19.11 & 18.80 \\
\hline $0.389[\mathrm{Ne} \mathrm{III}]+\mathrm{H} 7$ & $23.59 \pm 0.37$ & $25.99 \pm 0.82$ & $26.19 \pm 0.82$ & 23.38 & 23.35 \\
\hline $0.403 \mathrm{He} \mathrm{I}$ & $1.30 \pm 0.13$ & $1.40 \pm 0.20$ & $1.42 \pm 0.20$ & 1.72 & 1.66 \\
\hline 0.407 [S II] & $0.80 \pm 0.13$ & $0.86 \pm 0.16$ & $0.87 \pm 0.16$ & 0.12 & 0.13 \\
\hline $0.410 \mathrm{H} \delta$ & $24.19 \pm 0.37$ & $26.28 \pm 0.81$ & $25.78 \pm 0.80$ & 25.81 & 25.46 \\
\hline $0.434 \mathrm{H} \gamma$ & $45.88 \pm 0.67$ & $48.36 \pm 1.12$ & $47.79 \pm 1.11$ & 47.29 & 47.09 \\
\hline $0.436[\mathrm{O}$ III $]$ & $9.82 \pm 0.17$ & $10.23 \pm 0.48$ & $10.45 \pm 0.48$ & 7.98 & 7.87 \\
\hline $0.447 \mathrm{He} \mathrm{I}$ & $3.72 \pm 0.12$ & $3.84 \pm 0.29$ & $3.93 \pm 0.30$ & 3.57 & 3.45 \\
\hline 0.469 He II & $2.20 \pm 0.08$ & $2.22 \pm 0.22$ & $2.29 \pm 0.22$ & 0.00 & 2.21 \\
\hline $0.471[\mathrm{Ar}$ IV $]+\mathrm{He} \mathrm{I}$ & $1.29 \pm 0.08$ & $1.30 \pm 0.17$ & $1.34 \pm 0.17$ & 1.00 & 1.03 \\
\hline $0.474[\mathrm{Ar}$ IV $]$ & $1.18 \pm 0.07$ & $1.19 \pm 0.16$ & $1.22 \pm 0.16$ & 0.37 & 0.40 \\
\hline $0.486 \mathrm{H} \beta$ & $100.00 \pm 1.44$ & $100.00 \pm 1.46$ & $100.00 \pm 1.46$ & 100.00 & 100.00 \\
\hline $0.492 \mathrm{He} \mathrm{I}$ & $1.08 \pm 0.09$ & $1.07 \pm 0.16$ & $1.11 \pm 0.16$ & 0.95 & 0.92 \\
\hline $0.496[\mathrm{O}$ III $]$ & $101.28 \pm 1.46$ & $100.12 \pm 1.72$ & $103.51 \pm 1.77$ & 89.07 & 86.16 \\
\hline $0.501[\mathrm{O}$ III $]$ & $304.90 \pm 4.36$ & $300.32 \pm 4.34$ & $310.74 \pm 4.58$ & 268.10 & 259.34 \\
\hline 0.588 He I & $11.76 \pm 0.19$ & $11.02 \pm 0.47$ & $11.53 \pm 0.49$ & 9.02 & 8.70 \\
\hline $0.656 \mathrm{H} \alpha$ & $300.67 \pm 4.29$ & $273.73 \pm 4.38$ & $273.80 \pm 4.38$ & 282.51 & 284.85 \\
\hline $0.658[\mathrm{~N} \mathrm{II}]$ & $0.81 \pm 0.04$ & $0.74 \pm 0.12$ & $0.78 \pm 0.13$ & 0.65 & 0.70 \\
\hline 0.668 He I & $3.07 \pm 0.06$ & $2.78 \pm 0.23$ & $2.93 \pm 0.24$ & 2.44 & 2.35 \\
\hline $0.672[\mathrm{~S}$ II $]$ & $2.39 \pm 0.06$ & $2.16 \pm 0.21$ & $2.27 \pm 0.21$ & 0.63 & 0.80 \\
\hline $0.673[\mathrm{~S}$ II] & $2.12 \pm 0.05$ & $1.92 \pm 0.19$ & $2.02 \pm 0.20$ & 0.48 & 0.60 \\
\hline $0.707 \mathrm{He} \mathrm{I}$ & $5.21 \pm 0.09$ & $4.64 \pm 0.30$ & $4.91 \pm 0.31$ & 3.30 & 3.12 \\
\hline 0.714 [Ar III $]$ & $1.93 \pm 0.05$ & $1.71 \pm 0.18$ & $1.82 \pm 0.19$ & 2.07 & 1.98 \\
\hline $0.728 \mathrm{He} \mathrm{I}$ & $0.79 \pm 0.04$ & $0.70 \pm 0.12$ & $0.74 \pm 0.12$ & 0.69 & 0.66 \\
\hline $0.901 \mathrm{~Pa} 10$ & $1.39 \pm 0.07$ & $1.20 \pm 0.15$ & $1.28 \pm 0.16$ & 1.83 & 1.80 \\
\hline
\end{tabular}


Table 7-Continued

\begin{tabular}{|c|c|c|c|c|c|}
\hline \multirow[b]{2}{*}{ ION } & \multirow[b]{2}{*}{$100 \times F(\lambda) / F(\mathrm{H} \beta)^{\mathrm{a}}$} & \multirow[b]{2}{*}{$100 \times I(\lambda) / I(\mathrm{H} \beta)^{\mathrm{b}}$} & \multirow[b]{2}{*}{$100 \times I(\lambda) / I(\mathrm{H} \beta)^{\mathrm{c}}$} & \multicolumn{2}{|c|}{ CLOUDY } \\
\hline & & & & stars & stars+shock \\
\hline $0.907[\mathrm{~S}$ III $]$ & $3.66 \pm 0.09$ & $3.09 \pm 0.24$ & $3.30 \pm 0.25$ & 3.07 & 2.94 \\
\hline $0.953[\mathrm{~S} \mathrm{III}]$ & $8.17 \pm 0.22$ & $6.72 \pm 0.35$ & $7.24 \pm 0.37$ & 7.61 & 7.29 \\
\hline $0.955 \mathrm{~Pa} \epsilon$ & $3.86 \pm 0.19$ & $3.22 \pm 0.25$ & $3.45 \pm 0.27$ & 3.48 & 3.43 \\
\hline \multicolumn{6}{|c|}{ b) Near infrared spectrum } \\
\hline $0.953[\mathrm{~S}$ III $]$ & $8.17 \pm 0.58$ & $6.72 \pm 0.52$ & $7.24 \pm 0.55$ & 7.61 & 7.29 \\
\hline $0.955 \mathrm{~Pa} \epsilon$ & $4.33 \pm 0.36$ & $3.63 \pm 0.34$ & $3.89 \pm 0.36$ & 3.48 & 3.43 \\
\hline $1.005 \mathrm{~Pa} \delta$ & $5.99 \pm 0.41$ & $4.88 \pm 0.39$ & $5.25 \pm 0.41$ & 5.19 & 5.11 \\
\hline $1.083 \mathrm{He} \mathrm{I}$ & $32.78 \pm 2.14$ & $26.36 \pm 1.78$ & $28.53 \pm 1.93$ & 27.61 & 26.56 \\
\hline $1.091 \mathrm{He} \mathrm{I}$ & $0.49 \pm 0.06$ & $0.39 \pm 0.08$ & $0.42 \pm 0.09$ & 0.25 & 0.24 \\
\hline $1.094 \mathrm{~Pa} \gamma$ & $9.26 \pm 0.61$ & $7.47 \pm 0.54$ & $8.08 \pm 0.58$ & 8.29 & 8.17 \\
\hline $1.279 \mathrm{He} \mathrm{I}$ & $1.14 \pm 0.09$ & $0.90 \pm 0.13$ & $0.98 \pm 0.14$ & 0.46 & 0.44 \\
\hline $1.282 \mathrm{~Pa} \beta$ & $15.75 \pm 1.03$ & $12.40 \pm 0.86$ & $13.49 \pm 0.93$ & 14.81 & 14.72 \\
\hline 1.641 Br12 & $0.63 \pm 0.05$ & $0.50 \pm 0.09$ & $0.54 \pm 0.10$ & 0.54 & 0.53 \\
\hline $1.643[\mathrm{Fe} \mathrm{II}]$ & $0.27 \pm 0.04$ & $0.21 \pm 0.07$ & $0.23 \pm 0.08$ & 0.31 & 0.82 \\
\hline $1.681 \mathrm{Br} 11$ & $0.64 \pm 0.05$ & $0.51 \pm 0.09$ & $0.55 \pm 0.10$ & 0.67 & 0.66 \\
\hline 1.736 $\operatorname{Br} 10$ & $0.97 \pm 0.07$ & $0.75 \pm 0.11$ & $0.82 \pm 0.12$ & 0.86 & 0.85 \\
\hline $1.876 \mathrm{~Pa} \alpha$ & $37.39 \pm 2.52$ & $28.42 \pm 2.00$ & $31.15 \pm 2.19$ & 29.11 & 29.05 \\
\hline $1.944 \mathrm{Br} \delta$ & $2.42 \pm 0.17$ & $1.84 \pm 0.19$ & $2.02 \pm 0.20$ & 1.62 & 1.59 \\
\hline $2.058 \mathrm{He} \mathrm{I}$ & $1.76 \pm 0.12$ & $1.33 \pm 0.15$ & $1.46 \pm 0.17$ & 1.37 & 1.30 \\
\hline $2.122 \mathrm{H}_{2} 1-0 \mathrm{~S}(1)$ & $0.21 \pm 0.02$ & $0.16 \pm 0.05$ & $0.17 \pm 0.06$ & $\cdots$ & $\cdots$ \\
\hline $2.165 \mathrm{Br} \gamma$ & $3.60 \pm 0.24$ & $2.72 \pm 0.24$ & $2.99 \pm 0.26$ & 2.40 & 2.36 \\
\hline $2.248 \mathrm{H}_{2} 2-1 \mathrm{~S}(1)$ & $0.14 \pm 0.04$ & $0.11 \pm 0.08$ & $0.11 \pm 0.08$ & $\cdots$ & $\cdots$ \\
\hline
\end{tabular}

${ }^{\mathrm{a}} F(\mathrm{H} \beta)=(6.672 \pm 0.012) \times 10^{-14} \mathrm{erg} \mathrm{s}^{-1} \mathrm{~cm}^{-2}$.

${ }^{\mathrm{b}}$ Corrected for extinction with $C(\mathrm{H} \beta)=0.135$ and $\mathrm{EW}(\mathrm{abs})=0.5 \AA$.

${ }^{\mathrm{c}}$ Corrected for extinction with $C(\mathrm{H} \beta)=0.105$ and $\mathrm{EW}(\mathrm{abs})=0.35 \AA$. 
Table 8. Extinction-corrected fluxes of strong hydrogen lines

\begin{tabular}{crrrrrr}
\hline \hline & \multicolumn{7}{c}{ Galaxy } & \\
\cline { 2 - 5 } Line & II Zw 40 & Mrk 71 & Mrk 930 & Mrk 996 & SBS 0335-052E & Theor. $^{\text {a }}$ \\
\hline \multicolumn{7}{c}{ a) Optical spectrum } \\
$0.410 \mathrm{H} \delta$ & 32.0 & 26.8 & 26.8 & 29.5 & 25.8 & 26.2 \\
$0.434 \mathrm{H} \gamma$ & 48.9 & 48.7 & 46.3 & 54.9 & 47.8 & 47.3 \\
$0.486 \mathrm{H} \beta$ & 100.0 & 100.0 & 100.0 & 100.0 & 100.0 & 100.0 \\
$0.656 \mathrm{H} \alpha$ & 283.9 & 278.7 & 284.1 & 291.2 & 273.8 & 279.0 \\
& & \multicolumn{7}{c}{ b) NIR spectrum } & & \\
$1.094 \mathrm{P} \gamma$ & 7.9 & 9.4 & 9.2 & 8.3 & 8.1 & 8.6 \\
$1.282 \mathrm{P} \beta$ & 13.2 & 15.9 & 15.8 & 14.8 & 13.5 & 15.2 \\
$2.165 \mathrm{Br} \gamma$ & 2.8 & 2.4 & 2.5 & 2.7 & 3.0 & 2.5 \\
\hline
\end{tabular}

${ }^{\text {a Recombination }} \underset{\text { Hummer \& Storey }}{(1987)}$.
(19tios

Table 9. Extinction coefficients $C(\mathrm{H} \beta)$ derived from individual hydrogen line to $\mathrm{H} \beta$ flux ratios $^{a}$

\begin{tabular}{cccccc}
\hline \hline & \multicolumn{5}{c}{ Galaxy } \\
\cline { 2 - 6 } Line & II Zw 40 & Mrk 71 & Mrk 930 & Mrk 996 & SBS 0335-052E \\
\hline & & \multicolumn{5}{c}{ a) Optical spectrum } \\
$0.410 \mathrm{H} \delta$ & $0.686 \pm 0.215$ & $0.112 \pm 0.150$ & $0.348 \pm 0.287$ & $0.188 \pm 0.224$ & $0.051 \pm 0.159$ \\
$0.434 \mathrm{H} \gamma$ & $1.001 \pm 0.247$ & $0.091 \pm 0.216$ & $0.416 \pm 0.317$ & $0.005 \pm 0.244$ & $0.026 \pm 0.221$ \\
$0.656 \mathrm{H} \alpha$ & $1.181 \pm 0.115$ & $0.217 \pm 0.112$ & $0.348 \pm 0.129$ & $0.403 \pm 0.114$ & $0.093 \pm 0.112$ \\
& & & b) NIR spectrum & \\
$1.094 \mathrm{P} \gamma$ & $1.066 \pm 0.180$ & $0.231 \pm 0.180$ & $0.341 \pm 0.069$ & $0.381 \pm 0.184$ & $0.048 \pm 0.217$ \\
$1.282 \mathrm{P} \beta$ & $1.036 \pm 0.164$ & $0.200 \pm 0.163$ & $0.331 \pm 0.052$ & $0.387 \pm 0.166$ & $0.016 \pm 0.197$ \\
$2.165 \mathrm{Br} \gamma$ & $1.167 \pm 0.139$ & $0.163 \pm 0.139$ & $0.305 \pm 0.136$ & $0.443 \pm 0.141$ & $0.175 \pm 0.168$ \\
\hline
\end{tabular}

aThe adopted theoretical recombination ratios are for $T_{e}=15000 \mathrm{~K}$ and $N_{e}=100 \mathrm{~cm}^{-3}$ from Hummer \& Storey (1987) (see Table 8). 
Table 10. $\mathrm{H}_{2}$ emission-line flux ratios

\begin{tabular}{|c|c|c|c|c|c|c|c|}
\hline \multirow[b]{2}{*}{ Line } & \multicolumn{5}{|c|}{ Galaxy } & \multicolumn{2}{|c|}{ Model $^{\mathrm{a}}$} \\
\hline & II Zw 40 & Mrk 71 & Mrk 930 & Mrk 996 & SBS 0335-052E & Fluor & Coll \\
\hline $1.162 \mathrm{H}_{2} 2-0 \mathrm{~S}(1)$ & $\cdots$ & $0.1 \pm 0.1$ & $\cdots$ & $\cdots$ & $\cdots$ & 0.4 & 0.0 \\
\hline $1.207 \mathrm{H}_{2} 3-1 \mathrm{~S}(2)$ & $0.2 \pm 0.1$ & $0.2 \pm 0.1$ & $\ldots$ & $\cdots$ & $\cdots$ & 0.2 & 0.0 \\
\hline $1.233 \mathrm{H}_{2} 3-1 \mathrm{~S}(1)$ & $0.4 \pm 0.2$ & $0.4 \pm 0.2$ & $\ldots$ & $\cdots$ & $\cdots$ & 0.5 & 0.0 \\
\hline $1.238 \mathrm{H}_{2} 2-0 \mathrm{Q}(1)$ & $0.6 \pm 0.3$ & $0.3 \pm 0.2$ & $\cdots$ & $\cdots$ & $\cdots$ & 0.4 & 0.0 \\
\hline $1.311 \mathrm{H}_{2} 4-2 \mathrm{~S}(1)$ & $0.4 \pm 0.2$ & $0.3 \pm 0.2$ & $\cdots$ & $\cdots$ & $\cdots$ & 0.4 & 0.0 \\
\hline $1.314 \mathrm{H}_{2} 3-1 \mathrm{Q}(1)$ & $0.4 \pm 0.2$ & $0.3 \pm 0.2$ & $\cdots$ & $\cdots$ & $\cdots$ & 0.6 & 0.0 \\
\hline $1.601 \mathrm{H}_{2} 6-4 \mathrm{Q}(1)$ & $0.3 \pm 0.1$ & $0.1 \pm 0.1$ & $\cdots$ & $\cdots$ & $\cdots$ & 0.4 & 0.0 \\
\hline $1.957 \mathrm{H}_{2} 1-0 \mathrm{~S}(3)$ & $0.7 \pm 0.2$ & $0.9 \pm 0.3$ & $\cdots$ & $0.4 \pm 0.1$ & $\cdots$ & $\cdots$ & $\cdots$ \\
\hline $2.034 \mathrm{H}_{2} 1-0 \mathrm{~S}(2)$ & $0.4 \pm 0.1$ & $0.2 \pm 0.1$ & $\cdots$ & $0.3 \pm 0.1$ & $\cdots$ & 0.5 & 0.3 \\
\hline $2.073 \mathrm{H}_{2} 2-1 \mathrm{~S}(3)$ & $0.3 \pm 0.1$ & $0.2 \pm 0.1$ & $\ldots$ & $\ldots$ & $\cdots$ & 0.2 & 0.0 \\
\hline $2.122 \mathrm{H}_{2} 1-0 \mathrm{~S}(1)$ & $1.0 \pm 0.2$ & $1.0 \pm 0.2$ & $1.0 \pm 0.2$ & $1.0 \pm 0.2$ & $1.0 \pm 0.4$ & 1.0 & 1.0 \\
\hline $2.223 \mathrm{H}_{2} 1-0 \mathrm{~S}(0)$ & $0.4 \pm 0.1$ & $0.5 \pm 0.2$ & $0.6 \pm 0.2$ & $\ldots$ & $\ldots$ & 0.6 & 0.3 \\
\hline $2.248 \mathrm{H}_{2} 2-1 \mathrm{~S}(1)$ & $0.6 \pm 0.2$ & $0.5 \pm 0.2$ & $0.5 \pm 0.1$ & $0.3 \pm 0.1$ & $0.6 \pm 0.4$ & 0.5 & 0.0 \\
\hline $2.355 \mathrm{H}_{2} 2-1 \mathrm{~S}(0)$ & $0.2 \pm 0.1$ & $0.2 \pm 0.2$ & $\ldots$ & $0.2 \pm 0.1$ & $\ldots$ & 0.3 & 0.0 \\
\hline
\end{tabular}

${ }^{a}$ Model values are from Black \& van Dishoeck (1987). We adopt their model 1 for fluorescent lines and model S1 for collisionally excited lines. 
Table 11. Input parameters for stellar photoionization CLOUDY models

\begin{tabular}{lccccc}
\hline \hline Parameter & II Zw 40 & Mrk 71 & Mrk 930 & Mrk 996 & SBS 0335-052E \\
\hline$Q(\mathrm{H})$ & 53.136 & 52.064 & 53.893 & 52.750 & 53.562 \\
$T_{e f f}, \mathrm{~K}$ & 50000 & 50000 & 50000 & 50000 & 50000 \\
$N_{e}, \mathrm{~cm}^{-3}$ & 100 & 100 & 10 & & 100 \\
$\log f$ & -1.10 & -0.30 & -2.30 & -4.58 & -1.60 \\
$\log \mathrm{He} / \mathrm{H}$ & -1.07 & -1.07 & -1.05 & -0.93 & -1.09 \\
$\log \mathrm{C} / \mathrm{H}$ & -4.68 & -4.68 & -4.30 & -4.47 & -5.57 \\
$\log \mathrm{N} / \mathrm{H}$ & -5.29 & -5.83 & -5.34 & -4.90 & -6.13 \\
$\log \mathrm{O} / \mathrm{H}$ & -4.00 & -4.15 & -3.92 & -3.97 & -4.66 \\
$\log \mathrm{Ne} / \mathrm{H}$ & -4.71 & -4.87 & -4.70 & -4.67 & -5.40 \\
$\log \mathrm{Si} / \mathrm{H}$ & -5.35 & -5.35 & -5.35 & -5.57 & -6.05 \\
$\log \mathrm{S} / \mathrm{H}$ & -5.63 & -5.78 & -5.70 & -5.50 & -6.53 \\
$\log \mathrm{Cl} / \mathrm{H}$ & -7.49 & -7.59 & -7.49 & -7.47 & $\ldots$ \\
$\log \mathrm{Ar} / \mathrm{H}$ & -6.20 & -6.41 & -6.37 & -6.34 & -7.06 \\
$\log \mathrm{Fe} / \mathrm{H}$ & -5.80 & -5.90 & -5.99 & -5.70 & -6.10 \\
\hline
\end{tabular}

${ }^{a}$ For Mrk 996, a two-zone model for the electron number density spatial distribution has been adopted. For the inner high-excitation zone with radii $r$ between $0.8 \mathrm{pc}$ and $80 \mathrm{pc}$, the electron number density has a power-law dependence $N_{e} \sim r^{-2}$, decreasing from $4.5 \times 10^{6} \mathrm{~cm}^{-3}$ to $450 \mathrm{~cm}^{-3}$. For the lower-excitation outer zone with $r>80 \mathrm{pc}$, the electron number density has been set to a constant value of $450 \mathrm{~cm}^{-3}$. 
Table 12. Fluxes of NIR [Fe II] emission lines relative to $\mathrm{Br} \gamma$

\begin{tabular}{|c|c|c|c|c|c|}
\hline \multirow[b]{2}{*}{ ION } & \multirow[b]{2}{*}{$F(\lambda) / F(\operatorname{Br} \gamma)$} & \multirow[b]{2}{*}{$I(\lambda) / I(\operatorname{Br} \gamma)^{\mathrm{a}}$} & \multirow[b]{2}{*}{$I(\lambda) / I(\operatorname{Br} \gamma)^{\mathrm{b}}$} & \multicolumn{2}{|c|}{ CLOUDY } \\
\hline & & & & stars & stars+shock \\
\hline & \multicolumn{5}{|c|}{ a) II Zw 40} \\
\hline $1.257[\mathrm{Fe} \mathrm{II}]$ & 0.076 & 0.112 & 0.109 & 0.149 & $\cdots$ \\
\hline $1.643[\mathrm{Fe} \mathrm{II}]$ & 0.073 & 0.088 & 0.087 & 0.146 & $\cdots$ \\
\hline & \multicolumn{5}{|c|}{ b) Mrk 71} \\
\hline $1.257[\mathrm{Fe} \mathrm{II}]$ & 0.034 & 0.036 & 0.037 & 0.108 & 0.159 \\
\hline & \multicolumn{5}{|c|}{ c) Mrk 930} \\
\hline $1.257[\mathrm{Fe} \mathrm{II}]$ & 0.745 & 0.892 & 0.876 & 0.485 & 0.652 \\
\hline $1.643[\mathrm{Fe} \mathrm{II}]$ & 0.806 & 0.788 & 0.772 & 0.597 & 0.633 \\
\hline & \multicolumn{5}{|c|}{ d) Mrk 996} \\
\hline $1.257[\mathrm{Fe} \mathrm{II}]$ & 0.378 & 0.411 & 0.407 & 0.973 & $\ldots$ \\
\hline $1.643[\mathrm{Fe} \mathrm{II}]$ & 0.376 & 0.374 & 0.370 & 0.938 & $\ldots$ \\
\hline & \multicolumn{5}{|c|}{ e) SBS 0335-052E } \\
\hline $1.643[\mathrm{Fe} \mathrm{II}]$ & 0.075 & 0.077 & 0.077 & 0.121 & 0.347 \\
\hline
\end{tabular}

${ }^{\text {a }}$ Corrected for extinction derived from the observed Balmer decrement.

${ }^{\mathrm{b}}$ Corrected for extinction derived from the Balmer decrement, itself corrected for collisional excitation of hydrogen lines. 
Table 13. Fluxes of MIR emission lines

\begin{tabular}{|c|c|c|c|}
\hline \multirow[b]{2}{*}{ ION } & \multirow[b]{2}{*}{$100 \times I(\lambda) / I(\mathrm{H} \beta)$} & \multicolumn{2}{|c|}{ CLOUDY } \\
\hline & & stars & stars+shock \\
\hline & \multicolumn{3}{|c|}{ a) II $Z_{w} 40^{\mathrm{a}, b}$} \\
\hline $10.51[\mathrm{~S} \mathrm{IV}]$ & $40.77 \pm 0.88$ & 68.19 & $\cdots$ \\
\hline $12.81[\mathrm{Ne} \mathrm{II}]$ & $1.31 \pm 0.07$ & 0.43 & $\cdots$ \\
\hline 15.55 [Ne III] & $24.99 \pm 0.66$ & 33.56 & $\cdots$ \\
\hline 18.71 [S III] & $11.25 \pm 0.44$ & 10.40 & .. \\
\hline 25.99 [Fe II] & \multirow{2}{*}{\multicolumn{3}{|c|}{ b) Mrk $930^{\mathrm{c}, d}$}} \\
\hline & & & \\
\hline $12.81[\mathrm{Ne} \mathrm{II}]$ & $5.11 \pm 0.37$ & 3.14 & 3.35 \\
\hline 15.55 [Ne III] & $18.12 \pm 0.17$ & 27.82 & 28.06 \\
\hline 25.99 [Fe II] & \multicolumn{3}{|c|}{ c) $\operatorname{Mrk} 996^{e, f}$} \\
\hline $10.51[\mathrm{~S} \mathrm{IV}]$ & $5.15 \pm 0.02$ & 4.02 & $4.94^{\mathrm{g}}$ \\
\hline 12.81 [Ne II] & $5.76 \pm 0.05$ & 7.20 & $7.26^{\mathrm{g}}$ \\
\hline 15.55 [Ne III] & $16.32 \pm 0.76$ & 15.50 & $16.81^{\mathrm{g}}$ \\
\hline 18.71 [S III] & $11.21 \pm 0.76$ & 9.98 & $10.54^{\mathrm{g}}$ \\
\hline $25.89[\mathrm{O}$ IV $]$ & $1.82 \pm 0.45$ & 0.00 & $1.42^{\mathrm{g}}$ \\
\hline \multicolumn{4}{|c|}{ c) SBS $0335-052 \mathrm{E}^{\mathrm{h}, i}$} \\
\hline 10.51 [S IV] & $9.22 \pm 0.51$ & 5.33 & 5.27 \\
\hline 15.55 [Ne III] & $7.99 \pm 0.45$ & 7.52 & 7.28 \\
\hline
\end{tabular}

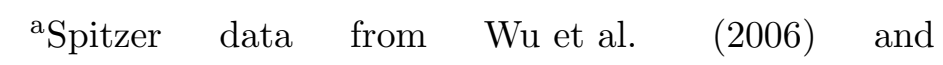
O'Halloran et al. (2006).

${ }^{b}$ Observed $\mathrm{H} \beta$ flux of $3.45 \times 10^{-13} \mathrm{erg} \mathrm{s}^{-1} \mathrm{~cm}^{-2}$ from Lagos et al. (2007).

'Spitzer data from O'Halloran et al. (2006).

${ }^{\mathrm{d}}$ Observed $\mathrm{H} \beta$ flux of $(2.54 \pm 0.10) \times 10^{-13} \mathrm{erg} \mathrm{s}^{-1} \mathrm{~cm}^{-2}$ from Moustakas \& Kennicutt (2006).

eSpitzer data from Thuan et al. (2008). 
${ }^{\mathrm{f}}$ Extinction-corrected $\mathrm{H} \beta$ flux of $6.6 \times 10^{-13} \mathrm{erg} \mathrm{s} \mathrm{s}^{-1}$ $\mathrm{cm}^{-2}$ from Thuan et al. (2008).

gModel D from Thuan et al. (2008).

${ }^{\mathrm{h}}$ Spitzer data from Houck et al. (2004).

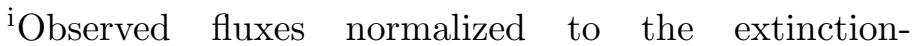
corrected $\mathrm{H} \beta$ flux of $(1.813 \pm 0.027) \times 10^{-13} \mathrm{erg} \mathrm{s}^{-1} \mathrm{~cm}^{-2}$ (Izotov et al. 2006b). 

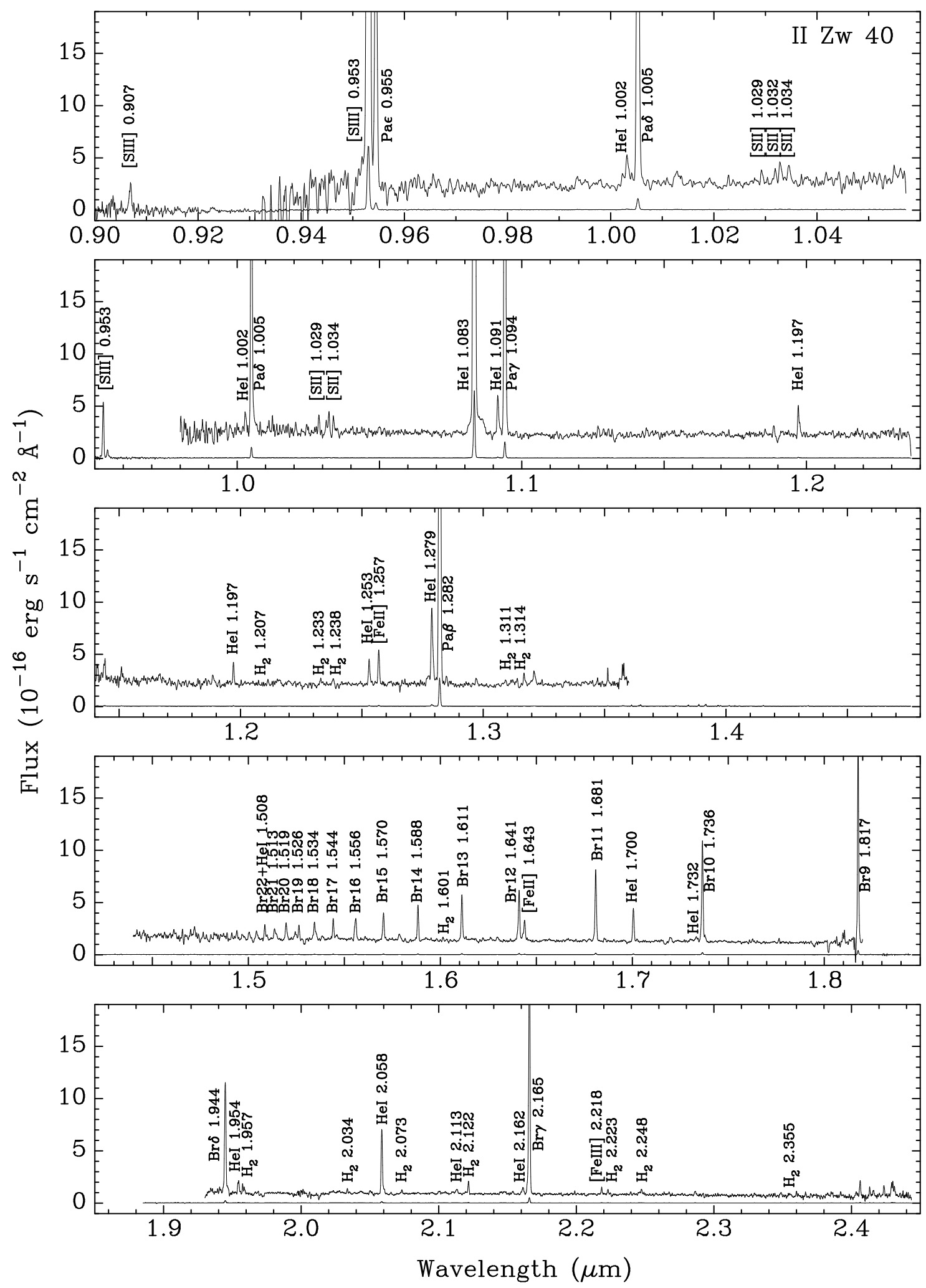

Fig. 1.- 3.5m APO/TripleSpec NIR spectrum of II Zw 40 in five orders. In each panel, the noisy regions of the upper spectrum are omitted. They are caused by insufficient sensitivity or strong telluric absorption. The flux scale on the y-axis corresponds to the upper spectrum. The lower spectrum is downscaled by a factor of 50 as compared to the upper spectrum. It is shown for the whole wavelength range in each order. 


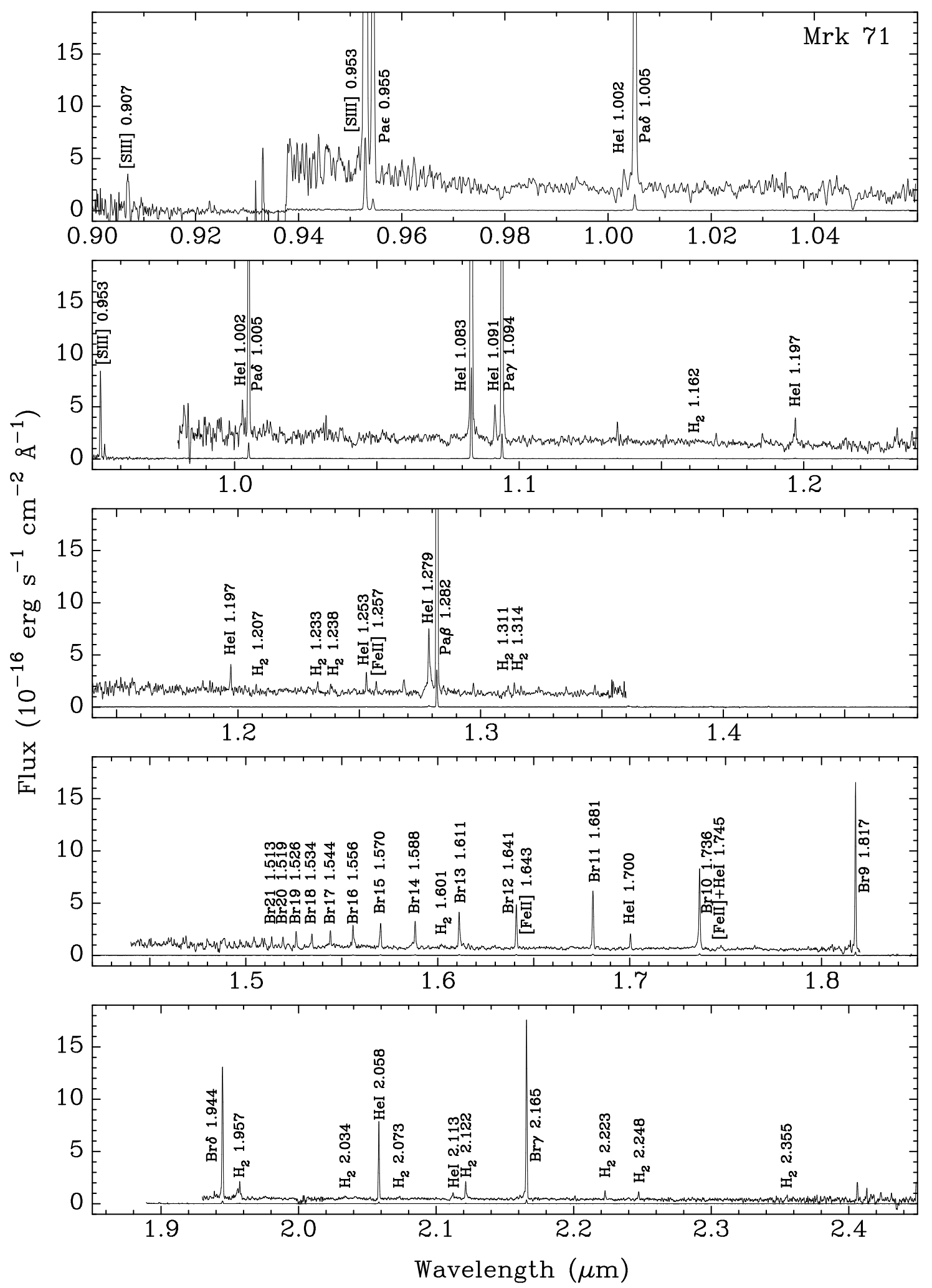

Fig. 2.- Same as in Fig. 1, but for Mrk 71. 


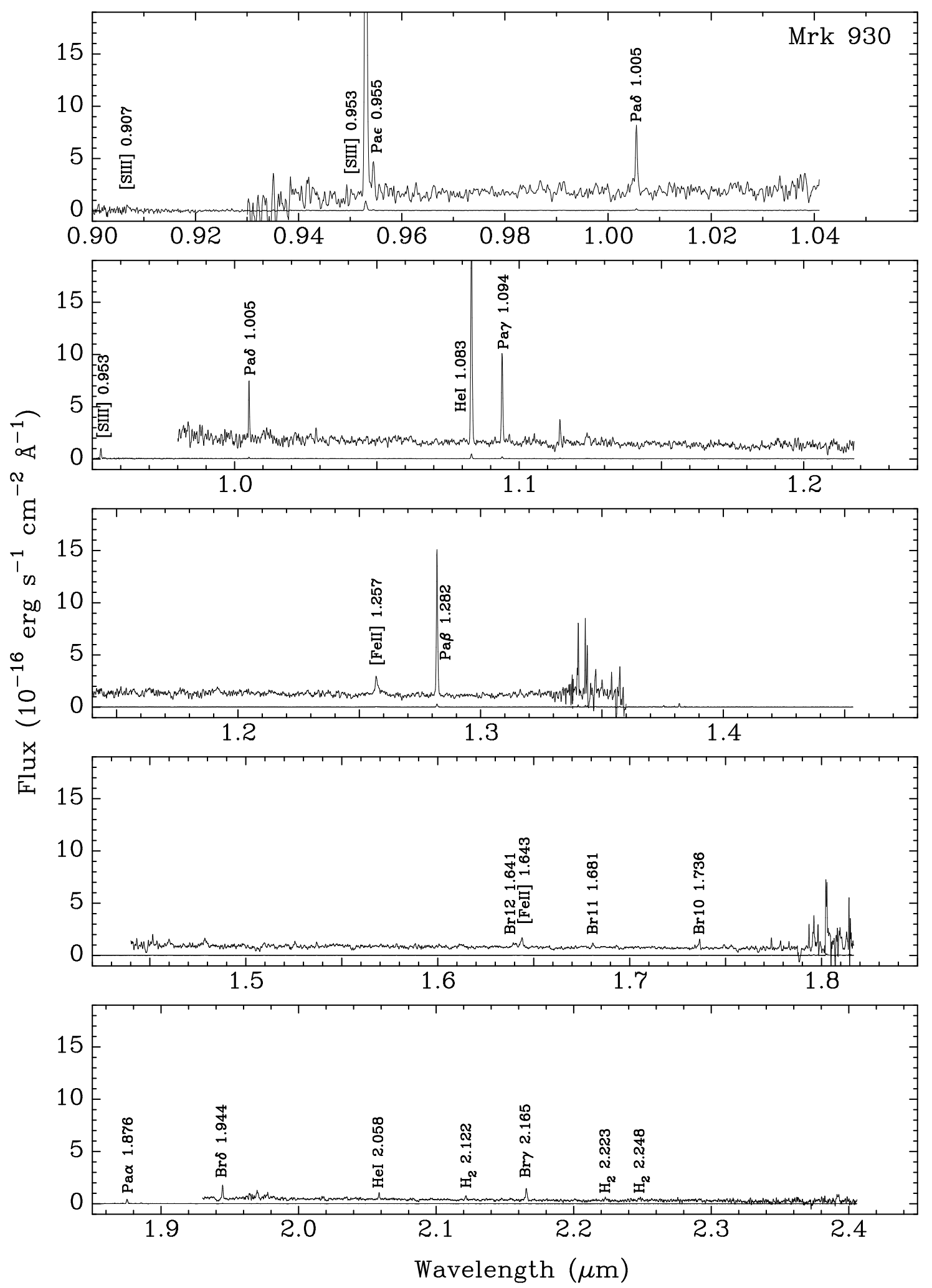

Fig. 3.- Same as in Fig. 1, but for Mrk 930. 


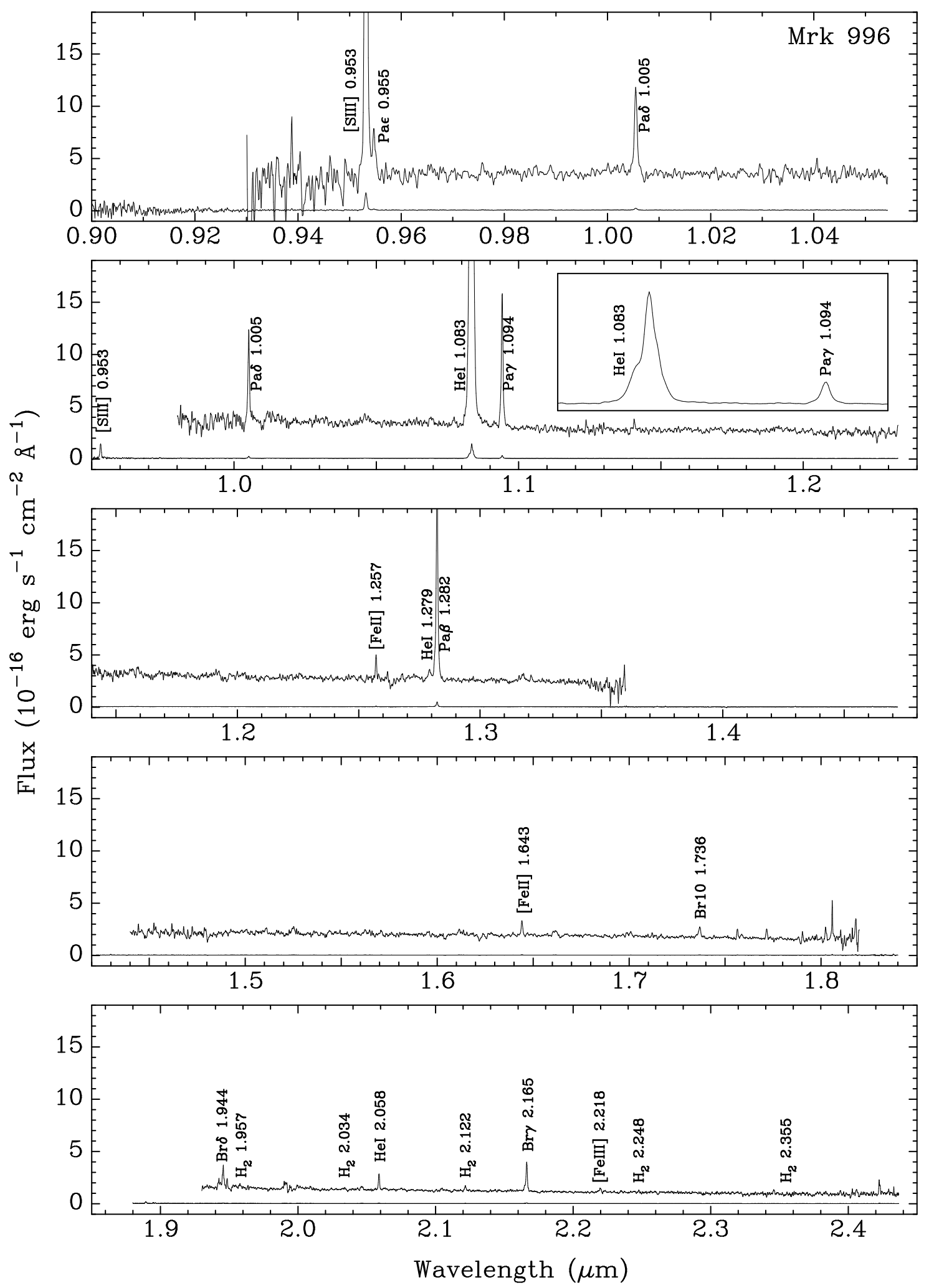

Fig. 4.- Same as in Fig. 1, but for Mrk 996. 


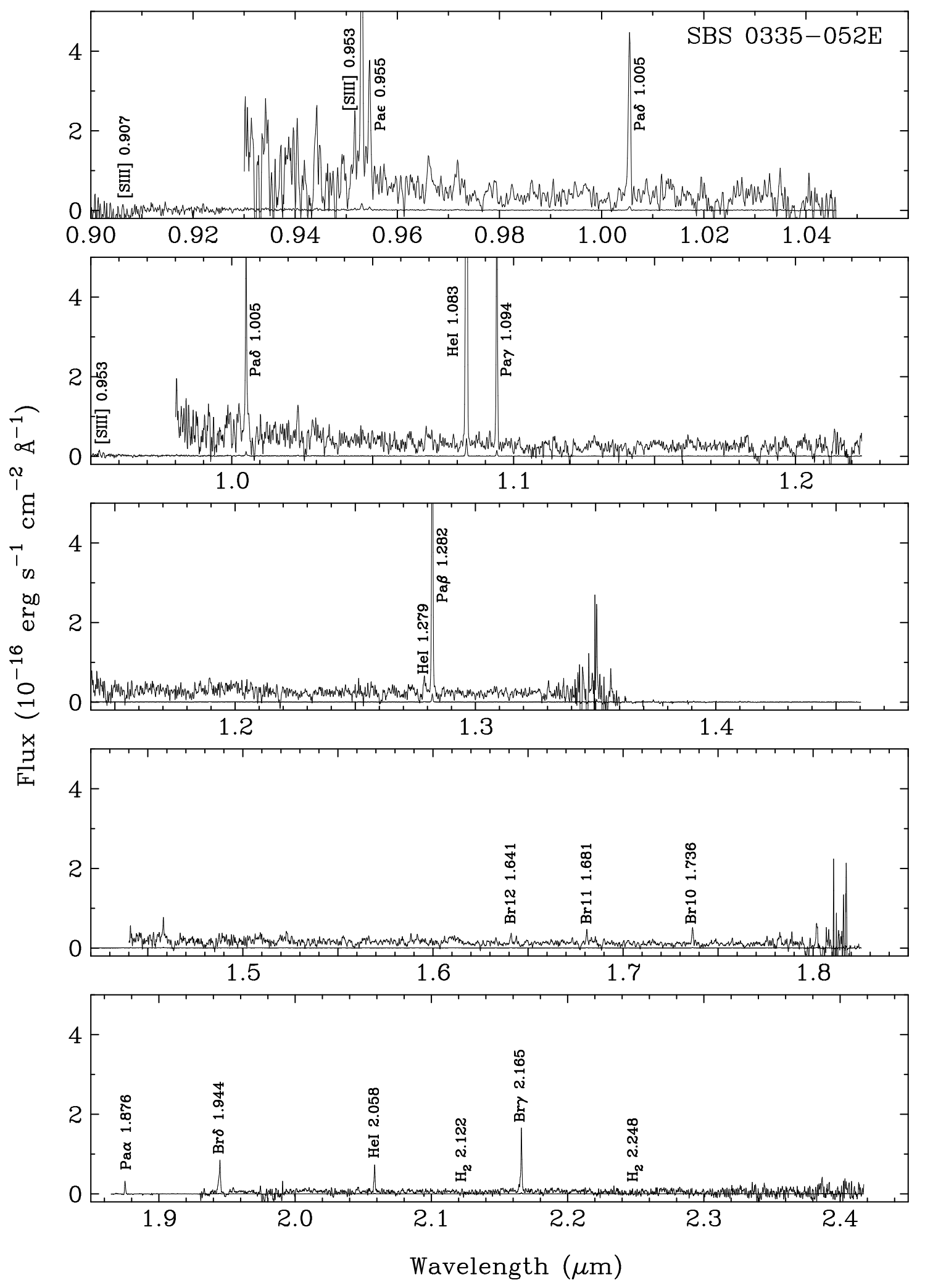

Fig. 5.- Same as in Fig. 1, but for SBS 0335-052E. 


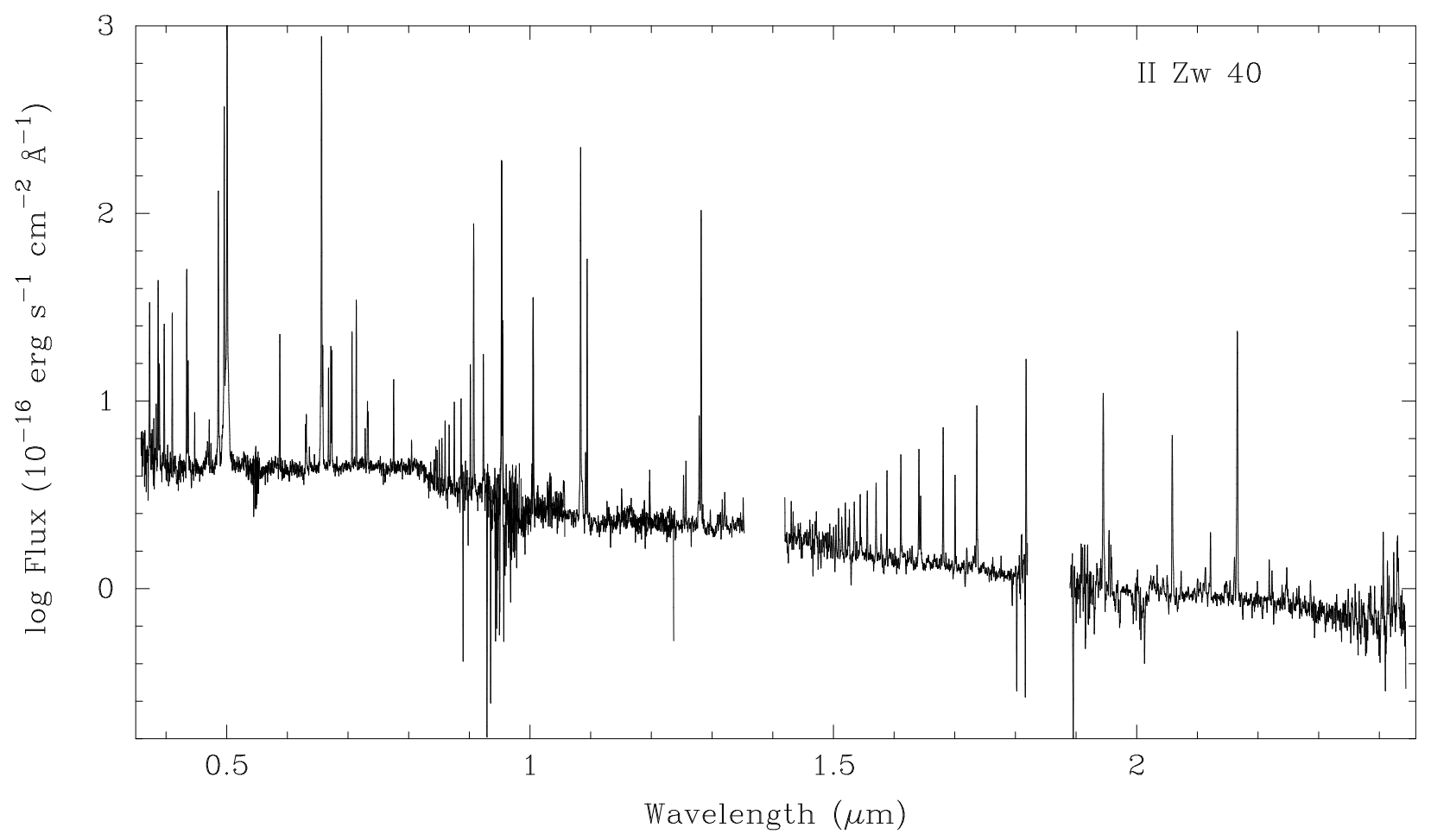

Fig. 6. - Redshift-corrected spectral energy distribution of II Zw 40 in the $0.36 \mu \mathrm{m}-2.4$ $\mu \mathrm{m}$ wavelength range 


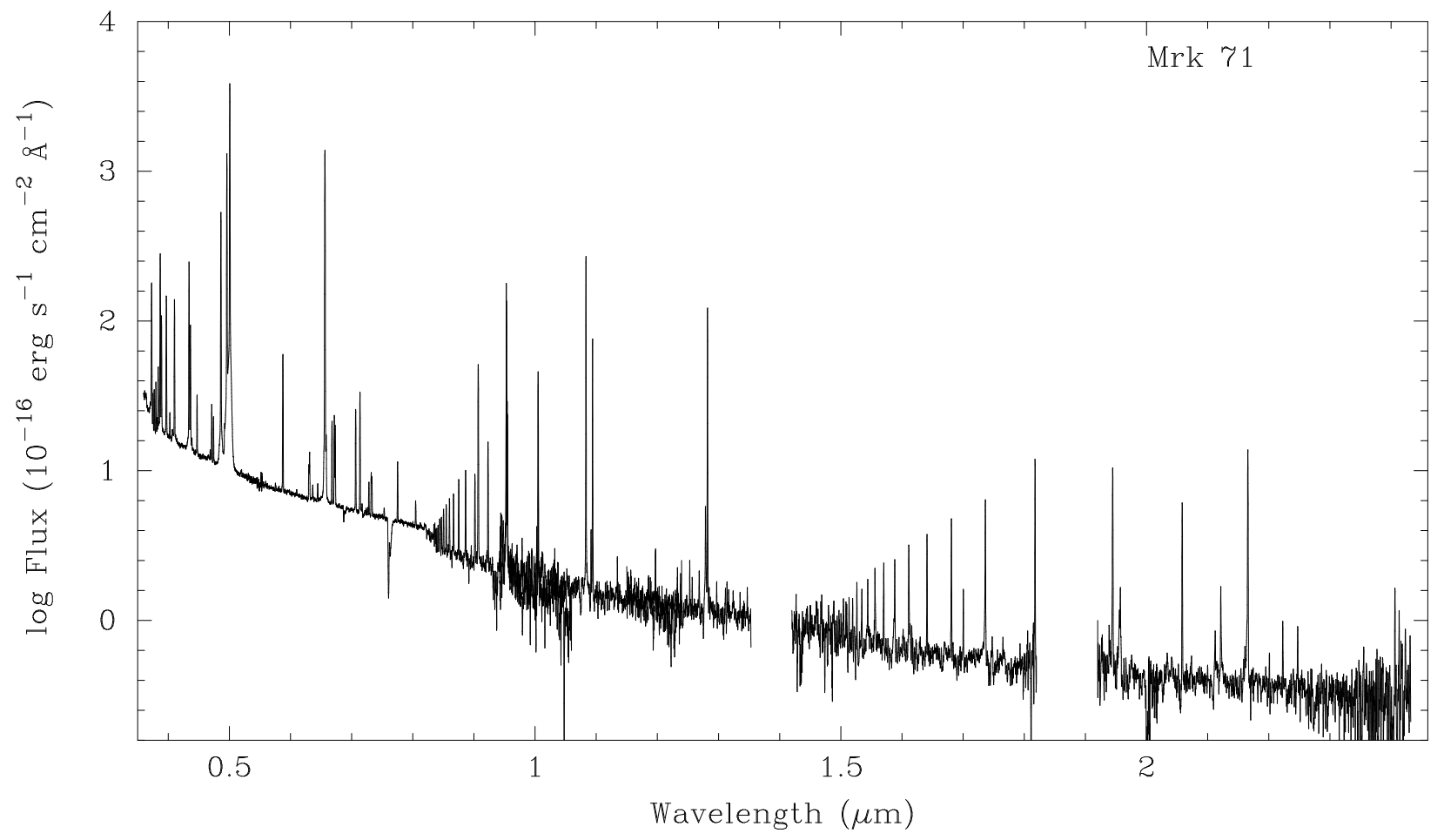

Fig. 7.- Same as in Fig. 6, but for Mrk 71. 


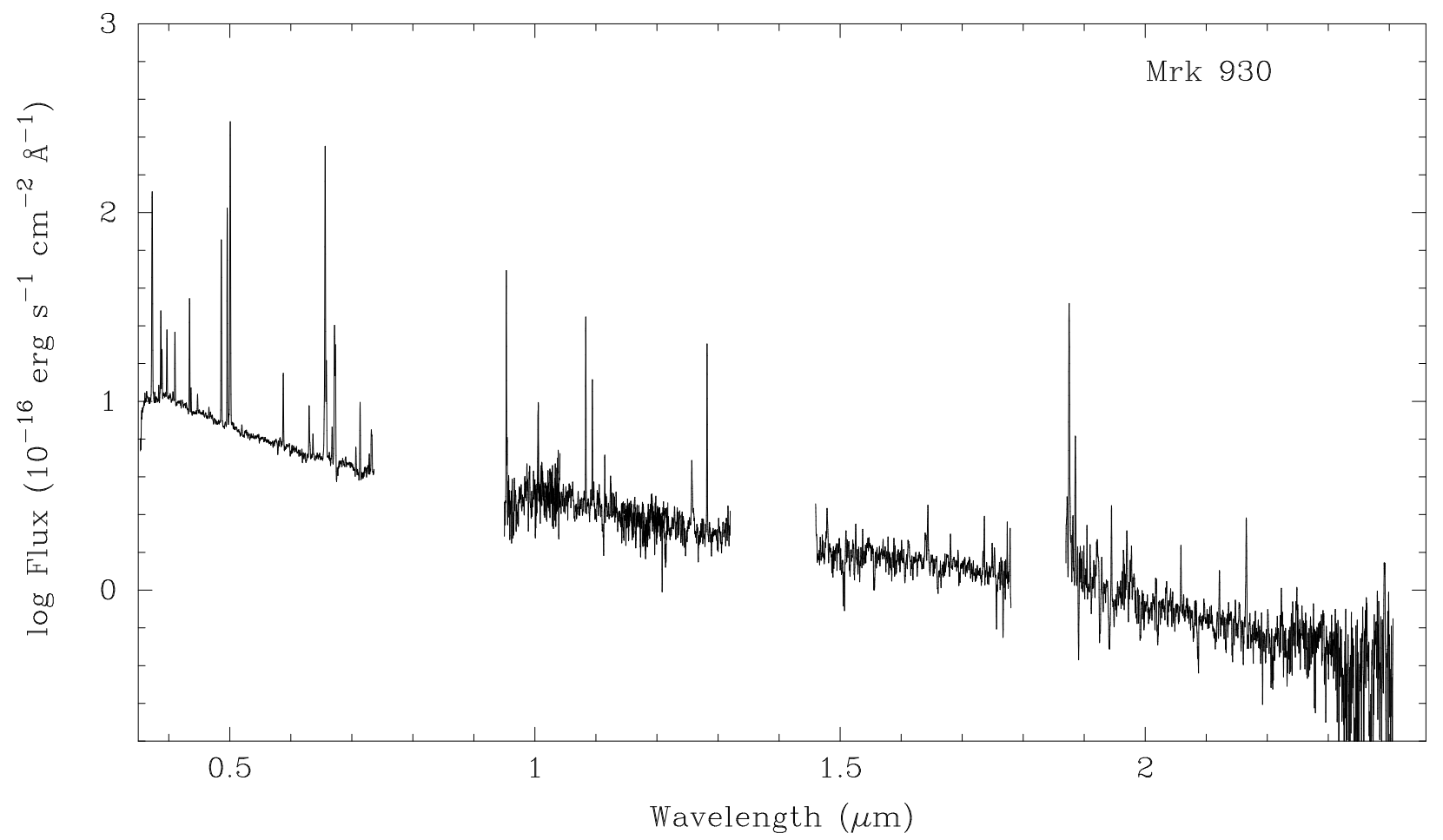

Fig. 8.- Same as in Fig. [6, but for Mrk 930. 


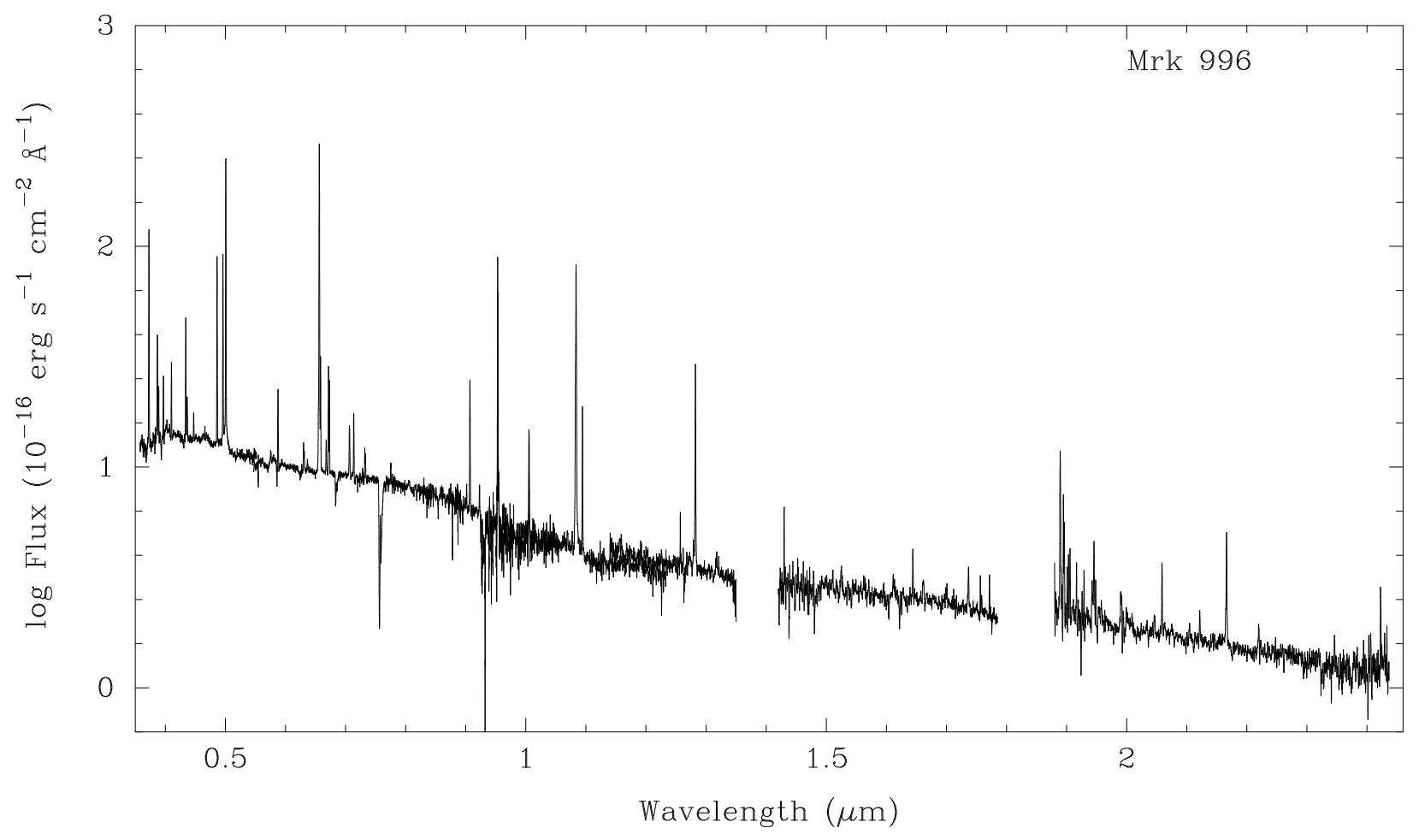

Fig. 9.- Same as in Fig. [6, but for Mrk 996. 


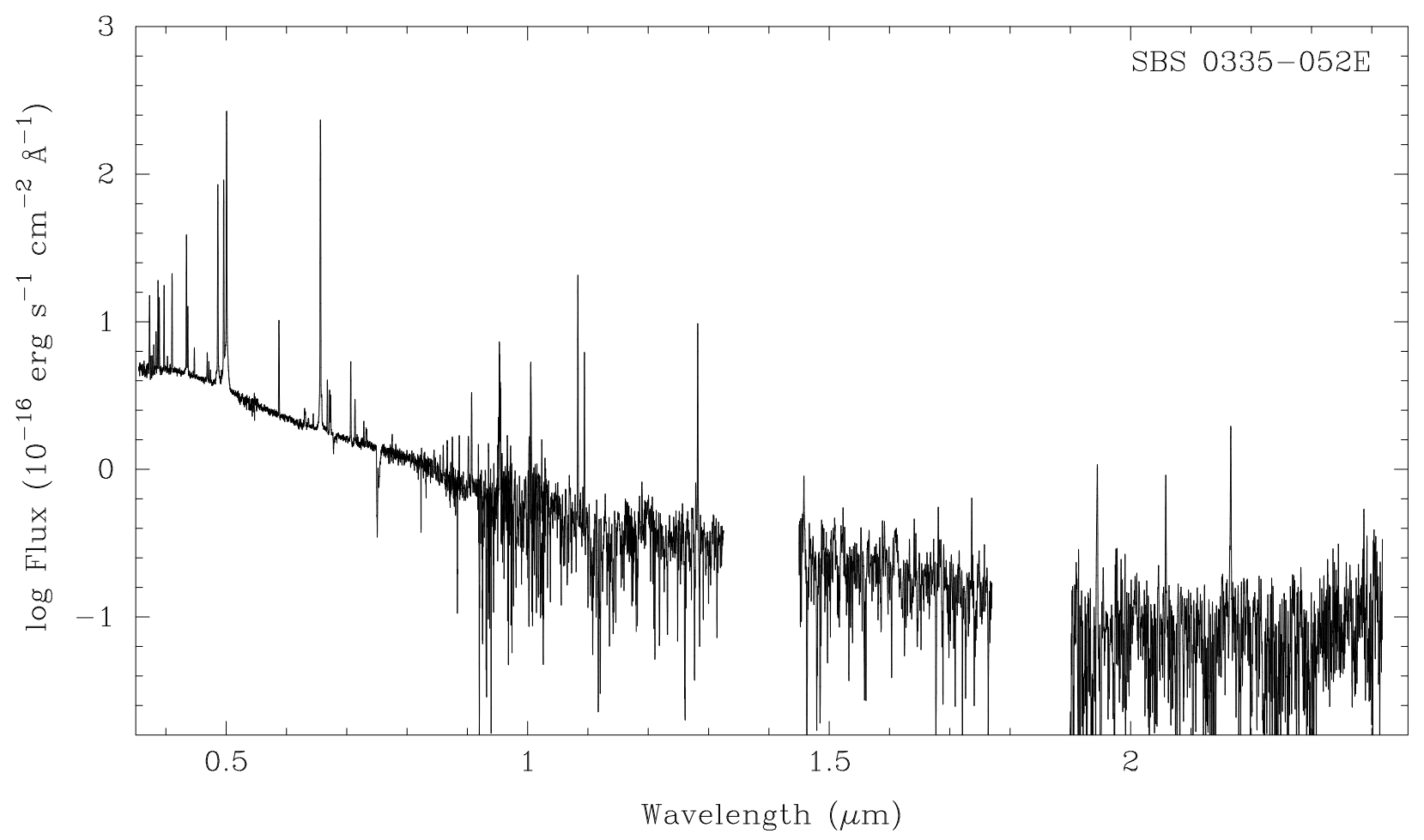

Fig. 10.- Same as in Fig. 6, but for SBS 0335-052E. 